\title{
Enumeration of ribbon 2-knots presented by virtual arcs with up to four crossings
}

\section{Taizo Kanenobu and Seiya Komatsu}

\begin{tabular}{|c|l|}
\hline Citation & OCAMI Preprint Series \\
\hline Issue Date & 2016 \\
\hline Type & Preprint \\
\hline Textversion & Author \\
\hline Rights & $\begin{array}{l}\text { C } 2017 \text { World Scientific Publishing Company. For personal use only. No other } \\
\text { uses without permission. }\end{array}$ \\
\hline Relation & $\begin{array}{l}\text { Preprint of an article submitted for consideration in Journal of Knot Theory and } \\
\text { Its Ramifications } \mathbb{C} 2017 \text { World Scientific Publishing Company. } \\
\text { https://www.worldscientific.com/worldscinet/jktr . }\end{array}$ \\
\hline Is version of & $\underline{\text { https://doi.org/10.1142/S0218216517500420 }}$ \\
\hline
\end{tabular}

From: Osaka City University Advanced Mathematical Institute http://www.sci.osaka-cu.ac.jp/OCAMI/publication/preprint/preprint.html 


\title{
Enumeration of ribbon 2-knots presented by virtual arcs with up to four crossings
}

\author{
Taizo Kanenobu and Seiya Komatsu \\ Department of Mathematics, Osaka City University, \\ Sugimoto, Sumiyoshi-ku, Osaka 558-8585, Japan \\ kanenobu@sci.osaka-cu.ac.jp
}

\begin{abstract}
We enumerate ribbon 2-knots presented by virtual arc diagrams with up to four classical crossings. We use a linear Gauss diagram for a virtual arc diagram.

Keywords: Ribbon 2-knot; virtual arc; welded arc; linear Gauss diagram; ribbon handlebody.

Mathematics Subject Classification 2000: 57Q45
\end{abstract}

\section{Intoduction}

A ribbon 2-knot is a knotted 2-sphere in $\boldsymbol{R}^{4}$ that bounds a ribbon 3-disk, which is an immersed 3 -disk with only ribbon singularities. The ribbon crossing number of a ribbon 2-knot is the minimal number of the ribbon singularities of any ribbon 3-disk bounding the knot [24]. The enumeration of the classical knots are based on the crossing number of a knot diagram. So, it is natural to enumerate ribbon 2-knots based on the ribbon crossing number. Yasuda has listed ribbon 2-knots with ribbon crossing number up to four [22,23,25,26,27].

Moreover, a ribbon 2-knot is presented by a virtual arc diagram, which was introduced by Satoh [19]; a virtual arc diagram has classical and virtual crossings and a classical crossing corresponds to a ribbon singularity. If a ribbon 2-knot is presented by a virtual arc diagram with $n$ classical crossings, then its ribbon crossing number is at most $n$. In this paper we enumerate ribbon 2 -knots based on the virtual arc presentation. Our main result is the table of ribbon 2-knots which are presented by virtual arc diagram with up to 4 crossings (Tables 2, 3 and 4 ). Therefore, the ribbon 2-knots in our table are contained in that of Yasuda. However, in the course of this enumeration we discovered an oversight in Yasuda's table, that is, we found 6 ribbon 2-knots whose Alexander polynomials are not listed in Yasuda's table.

From a virtual arc diagram we may obtain a linear Gauss diagram, which is an 
immersing interval with the preimages of each double point connected with a chord equipped with the sign of the crossing. Then an oriented linear Gauss diagram determines an associated ribbon 2-knot (Proposition 3.1). Also there are several moves for a linear Gauss diagram which do not change the isotopy class of the associated ribbon 2-knot (Subsec. 3.2); they are induced from the Reidemeister moves (moves $A_{I}, A_{I I}, A_{I I I}$ ), welded moves (moves $D, E_{I I}$ ), negative-amphicheirality of a ribbon 2-knot (reversing move), and composition of knots (Eq. (3.1)). Furthermore, they yield some simplified moves (moves $\Omega_{i}, i=1, \ldots, 8$ ), which are useful for investigating the equivalence of Gauss diagrams.

Using these moves, we enumerate linear Gauss diagrams. First, we show a ribbon 2-knot presented by linear Gauss diagrams with up to 4 chords is presented by one of the 13 linear Gauss diagrams $\Gamma_{l}^{4}, 1 \leq l \leq 13$, given in Fig. 29 (Lemma 5.1). Then, for each diagram we examine the corresponding ribbon 2-knot, where we calculate the knot group and Alexander polynomial (Sects. 5 and 7). We apply the above mentioned moves for considering the equivalence. However, in some cases we consider a ribbon handlebody (Lemma 7.3) or a virtual arc diagram (Lemma 7.5).

This paper is organized as follows: In Sec. 2, we introduce a ribbon 2-knot and its virtual arc presentation, where we use a ribbon handlebody to present a ribbon 3-disk. In Sec. 3, we introduce a linear Gauss diagram which presents a virtual arc diagram and so a ribbon 2-knot. In Sec. 4 , we give our main result, tables of ribbon 2-knots presented by virtual arc diagrams with up to 4 crossings (Tables 2, 3 and 4). Secs. 5, 6, and 7 are devoted to the proof of our main result. Most of the results in this paper are from the master's thesis of one of the authors [14].

\section{Ribbon 2-Knot and Its Virtual Arc Presentation}

In this section, we introduce a ribbon knot, virtual arc, welded arc, and virtual arc presentation of a ribbon 2-knot.

\subsection{Ribbon knot}

A ribbon $(n+1)$-disk, $n \geq 1$, is an immersed $(n+1)$-disk $D^{n+1}$ into $\boldsymbol{R}^{n+2}$ with only transverse double points such that the singular set consists of ribbon singularities, that is, the preimage of each ribbon singularity consists of a properly embedded $n$-disk in $D^{n+1}$ and an embedded $n$-disk interior to $D^{n+1}$. An $n$-knot is a ribbon $n$-knot if it bounds a ribbon $(n+1)$-disk in $\boldsymbol{R}^{n+2}$.

The ribbon crossing number of a ribbon $n$-knot $K$ is the smallest number of the ribbon singularities of any ribbon $(n+1)$-disk bounding the knot $K$; cf. [24]. Yasuda $[22,23,25,26,27]$ enumerated ribbon 2-knots with ribbon crossing number up to four.

A ribbon handlebody [3] $H$ is a ribbon 2-disk, which is a 2-dimensional handlebody in $\boldsymbol{R}^{3}$ consisting of $(p+1)$ 0-handles $D_{1}, D_{2}, \ldots, D_{p+1}, p$ 1-handles $B_{1}$, $B_{2}, \ldots, B_{p}$ and no 2 -handle such that the preimage of each ribbon singularity con- 
sists of an arc in the interior of a 0 -handle and a cocore of a 1-handle. We put $\mathcal{D}=D_{1} \cup D_{2} \cup \cdots \cup D_{p+1}$ and $\mathcal{B}=B_{1} \cup B_{2} \cup \cdots \cup B_{p}$. Then the set of ribbon singularities in $H$ is the connected components of the intersection $\mathcal{D} \cap \mathcal{B}$. Note that in order to present a ribbon handlebody we may use a ribbon regular projection defined in [6, Sect. 1] or [7, Sect. 3], .

We define the associated ribbon 2-knot in $\boldsymbol{R}^{4}=\boldsymbol{R}^{3} \times \boldsymbol{R}$ as the ribbon 2-knot that bounds the immersed 3 -disk $\mathcal{D} \times[-2,2] \cup \mathcal{B} \times[-1,1]$. Conversely, for any ribbon 2 -knot $K$, there exists a ribbon handlebody whose associated ribbon 2-knot is $K$; see $[10,21]$. Thus we may represent a ribbon 2 -knot by a ribbon handlebody.

\subsection{Virtual arc and welded arc}

A virtual knot diagram is an immersed circle and a virtual arc diagram is an immersed interval in the plane $\boldsymbol{R}^{2}$ such that each crossing is either a classical crossing or a virtual crossing as shown in Fig. 1.

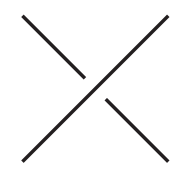

(a)

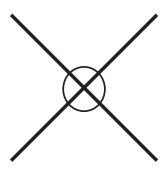

(b)

Fig. 1. (a) Classical crossing; (b) vitrtual crossing.

A virtual knot is an equivalence class of virtual knot diagram under the classical Reidemeister moves $A_{I}, A_{I I}, A_{I I I}$ as shown in Fig. 2 and the virtual Reidemeister moves $\mathrm{B}_{\mathrm{I}}, \mathrm{B}_{\mathrm{II}}, \mathrm{B}_{\mathrm{III}}, \mathrm{C}, \mathrm{E}_{\mathrm{I}}$ as shown in Fig. 3. Similarly, a virtual arc is an equivalence class of virtual arc diagram under the classical Reidemeister moves $A_{I}, A_{I I}, A_{\text {III }}$ and the virtual Reidemeister moves $\mathrm{B}_{\mathrm{I}}, \mathrm{B}_{\mathrm{II}}, \mathrm{B}_{\mathrm{III}}, \mathrm{C}$ as well as $\mathrm{E}_{\mathrm{I}}$ as shown in Fig. 3.

We introduce the welded moves $\mathrm{D}$ and $\mathrm{E}_{\mathrm{II}}$ as shown in Fig. 4; the move $\mathrm{D}$ is known as one of the "forbidden moves"; cf. [8,17]. Two virtual knot diagrams or virtual arc diagrams $A$ and $A^{\prime}$ are w-equivalent if there exist a finite sequence of the classical Reidemeister moves, virtual Reidemeister moves, and welded moves which transforms $A$ into $A^{\prime}$. A welded knot is an equivalence class under the wequivalence of virtual knot diagrams, and a welded arc is an equivalence class under the w-equivalence of virtual arc diagrams. A welded knot or arc is trivial if it is an equivalence class containing a virtual knot or virtual arc diagram without any crossing. Note that a welded knot and a welded arc are called a weakly virtual knot and a weakly virtual knot, respectively, in [9,19]. 


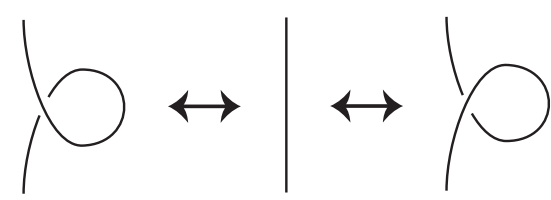

$\mathrm{A}_{\mathrm{I}}$
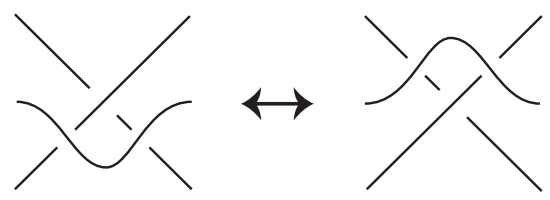
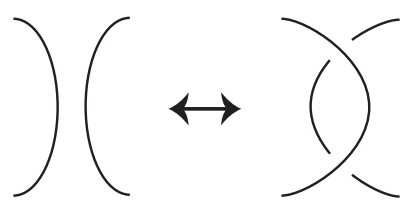

$\mathrm{A}_{\text {II }}$

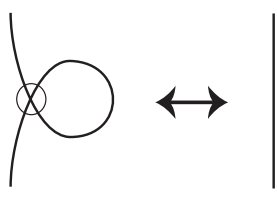

$\mathrm{B}_{\mathrm{I}}$
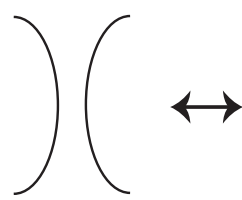

$\mathrm{B}_{\text {II }}$
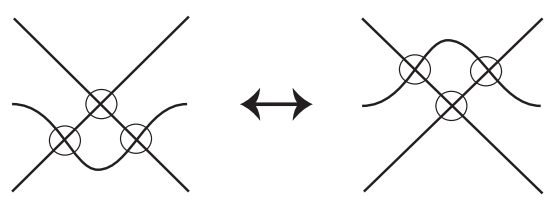

$\mathrm{B}_{\text {III }}$
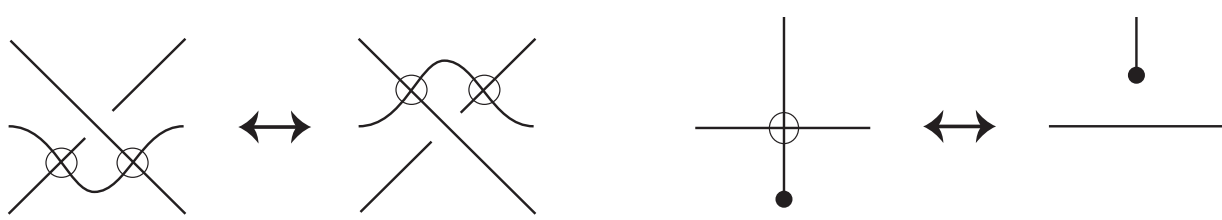

C

$\mathrm{E}_{\mathrm{I}}$

Fig. 3. Virtual Reidemeister moves $\mathrm{B}_{\mathrm{I}}, \mathrm{B}_{\mathrm{II}}, \mathrm{B}_{\mathrm{III}}, \mathrm{C}, \mathrm{E}_{\mathrm{I}}$.

\subsection{Virtual arc presentation of a ribbon 2-knot}

Given an oriented virtual arc diagram $A$, we can construct a ribbon handlebody $H_{A}$ as shown in Fig. 5, where an overpass at a classical crossing corresponds to a 0-handle containing a ribbon singularity, a virtual crossing corresponds to two 1- 

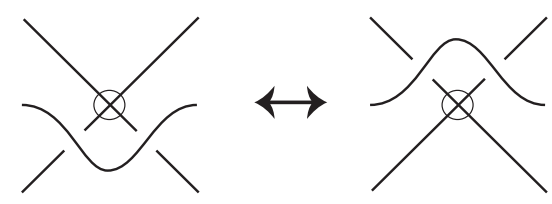

$\mathrm{D}$

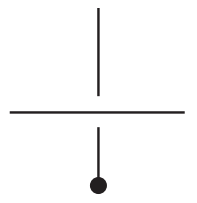

$\mathrm{E}_{\mathrm{II}}$

Fig. 4. Welded moves D, E $\mathrm{EII}_{\text {. }}$

handles passing over/under each other, and an end point corresponds to a 0-handle. Then we denote the associated ribbon 2-knot of $H_{A}$ by $\operatorname{Tube}(A)$. If two virtual arc diagrams $A$ and $A^{\prime}$ are w-equivalent, then the corresponding ribbon 2-knots Tube $(A)$ and Tube $\left(A^{\prime}\right)$ are ambient isotopic, and thus a welded arc determines a ribbon 2-knot. Conversely, any ribbon 2-knot is associated with some virtual arc diagram; see [19].

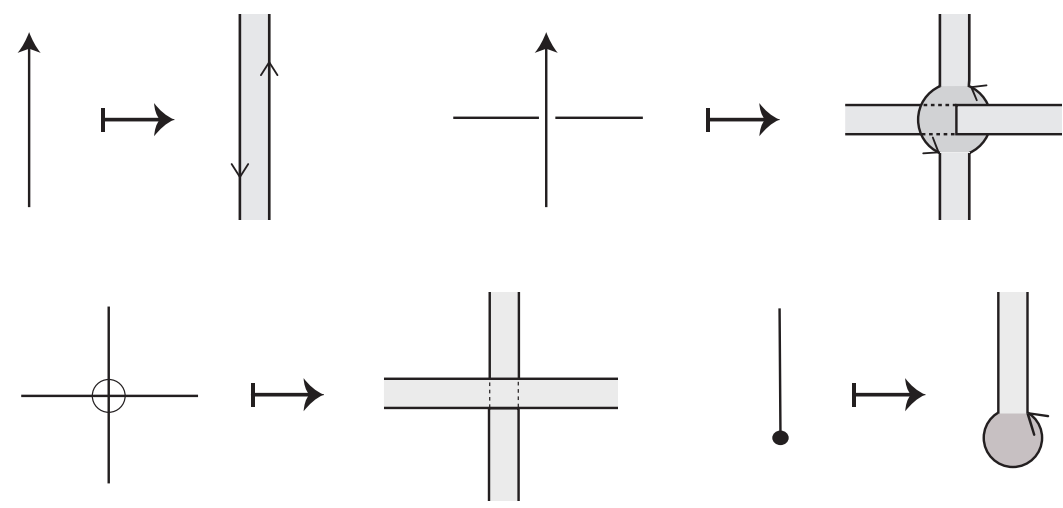

Fig. 5. Correspondence of an oriented virtual arc diagram to a ribbon handlebody.

Remark 2.1. Satoh [19] constructs the associated ribbon 2-knot Tube $(A)$ using a surface diagram, which is a projection image of a ribbon 2-knot in $\boldsymbol{R}^{4}$; see [1], cf. [10].

Furthermore, we define two oriented virtual arc diagrams $A$ and $A^{\prime}$ are $r$ equivalent, denoted by $A \stackrel{\mathrm{r}}{\sim} A^{\prime}$, if they correspond to the isotopic ribbon 2-knots.

Let $A$ be an oriented virtual arc diagram. We denote the horizontal mirror image by $A^{\dagger}$, which is obtained from $A$ by reflecting the diagram across a vertical plane. We denote the orientation-reversion of $A$ by $-A$. See Fig. 6; cf. [5, Sec. 2.2].

Then the both associated ribbon handlebodies $H_{A^{\dagger}}$ and $H_{-A}$ are obtained from $H_{A}$ by reflecting across the vertical plane and the projection plane, respectively. 


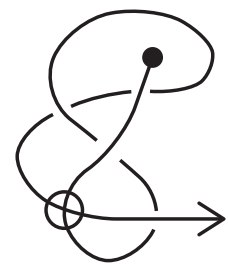

A

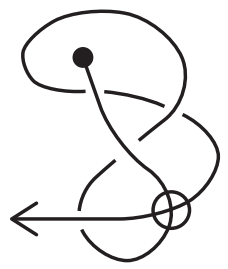

$A^{\dagger}$

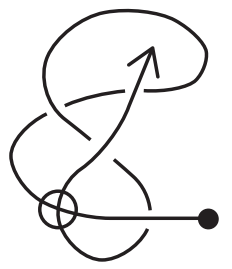

$-A$

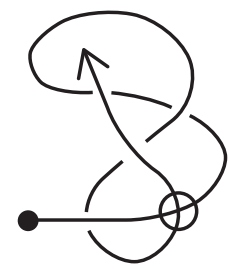

$-A^{\dagger}$

Fig. 6. Virtual arc diagrams $A, A^{\dagger},-A$, and $-A^{\dagger}$.

Alternatively, both $H_{A^{\dagger}}$ and $H_{-A}$ are obtained from $H_{A}$ by reversing the orientation of the immersed surface. This shows that a ribbon 2-knot is negative-amphicheiral; see [20, Theorem 2.18], [19, Proposition 4.1]. Thus we have:

Proposition 2.2. Let $A$ be an oriented virtual arc diagram. Then the associated ribbon 2-knots Tube $\left(A^{\dagger}\right)$ and Tube $(-A)$ are isotopic, which are the mirror images of Tube $(A)$. In other words, $A$ and $-A^{\dagger}$ are r-equivalent.

We define the product of two oriented virtual arc diagrams $A$ and $A^{\prime}$, denoted by $A \cdot A^{\prime}$, as follows: First, we deform the diagrams $A$ and $A^{\prime}$ so that the the terminal point of $A$ and the initial point of $A^{\prime}$ lie in the outermost region by the move $\mathrm{E}_{\mathrm{I}}$. We denote the resulting diagrams by $\tilde{A}$ and $\tilde{A}^{\prime}$. Then the product of $A$ and $A^{\prime}$ is obtained by connecting the terminal point of $\tilde{A}$ to the initial point of $\tilde{A}^{\prime}$ as shown in Fig. 7. It is easy to see that the product of oriented virtual arc diagrams is well defined up to w-equivalence.

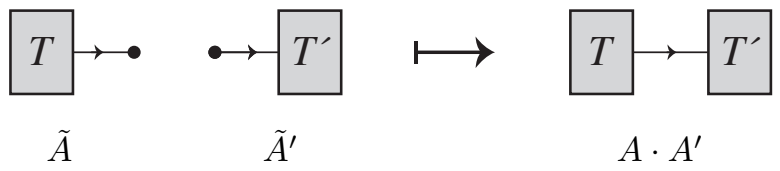

Fig. 7. Product of virtual arc diagrams.

The associated ribbon 2-knot $\operatorname{Tube}\left(A \cdot A^{\prime}\right)$ is the composition of $\operatorname{Tube}(A)$ and $\operatorname{Tube}\left(A^{\prime}\right) ; \operatorname{Tube}\left(A \cdot A^{\prime}\right)=\operatorname{Tube}(A) \# \operatorname{Tube}\left(A^{\prime}\right)$. Since the composition of oriented 2-knots is well defined up to isotopy, we have the following:

Proposition 2.3. Let $A$ and $A^{\prime}$ be oriented virtual arc diagrams. Then the associated ribbon 2-knots $\operatorname{Tube}\left(A \cdot A^{\prime}\right)$ and $\operatorname{Tube}\left(A^{\prime} \cdot A\right)$ are isotopic, $A \cdot A^{\prime} \stackrel{\mathrm{r}}{\sim} A^{\prime} \cdot A$.

Note that $A \cdot A^{\prime}$ and $A^{\prime} \cdot A$ may not be w-equivalent. We can see Proposition 2.3 by the associated ribbon handlebody as follows: $H_{A \cdot A^{\prime}}$ is the boundary connected 
sum of $H_{A}$ and $H_{A^{\prime}}$. Then by sliding $H_{A}$ along the boundary of $H_{A^{\prime}}$ we can isotope $H_{A \cdot A^{\prime}}$ into $H_{A^{\prime} \cdot\left(-A^{\dagger}\right)}$. This implies $\operatorname{Tube}\left(A \cdot A^{\prime}\right)$ is isotopic to Tube $\left(A^{\prime} \cdot\left(-A^{\dagger}\right)\right)$. From this we have further Tube $\left(A^{\prime} \cdot\left(-A^{\dagger}\right)\right)$ is isotopic to Tube $\left(\left(-A^{\dagger}\right) \cdot\left(-A^{\prime \dagger}\right)\right)$. Since the virtual arc $\left(-A^{\dagger}\right) \cdot\left(-A^{\prime \dagger}\right)$ is the same as $-\left(A^{\prime} \cdot A\right)^{\dagger}$, by Proposition 2.2 Tube $\left(\left(-A^{\dagger}\right) \cdot\left(-A^{\prime \dagger}\right)\right)$ is isotopic to Tube $\left(A^{\prime} \cdot A\right)$, completing the proof.

\section{Gauss diagram}

In this section, we first review a Gauss diagram of a virtual knot or a welded knot, and then introduce a linear Gauss diagram of a virtual arc. We give several moves of a linear Gauss diagram which do not change the isotopy class of the associated ribbon 2-knot.

\subsection{Gauss diagrams of virtual and welded knots}

The Gauss diagram of an oriented virtual knot diagram is an oriented circle as the preimage of the immersed circle with chords connecting the preimages of each classical crossing. We specify crossing information on each chord by directing the chord toward the under crossing equipped with the sign of the crossing. We call such a Gauss diagram a circular Gauss diagram. A circular Gauss diagram is considered up to orientation preserving homeomorphism of the underlying oriented circle. A circular Gauss diagram determines a virtual knot diagram up to virtual Reidemeister moves; see [2, Theorem 1.A]; cf. [12]. Namely, if two virtual knot diagrams have the same circular Gauss diagram, then there exist a finite sequence of virtual Reidemeister moves $\mathrm{B}_{\mathrm{I}}, \mathrm{B}_{\mathrm{II}}, \mathrm{B}_{\mathrm{III}}, \mathrm{C}$ which transforms one into the other.

The classical Reidemeister moves on a knot give rise to moves on circular Gauss diagrams. Depending on orientations of strings involved in the Reidemeister moves, one may distinguish four different versions of each of the $A_{I}$ and $A_{I I}$ moves, and eight versions of the $A_{\text {III }}$ move as shown in Figs. 8-10 [18, Figs. 2-4].

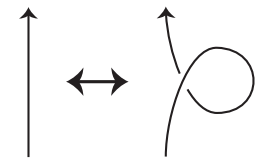

$\mathrm{A}_{\text {Ia }}$

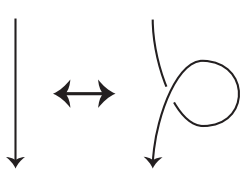

$\mathrm{A}_{\mathrm{Ib}}$

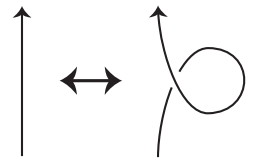

$\mathrm{A}_{\text {Ic }}$

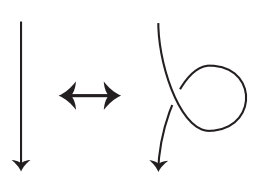

$\mathrm{A}_{\text {Id }}$

Fig. 8. Oriented Reidemeister moves of type I.

We give the corresponding moves of circular Gauss diagrams in Figs. 11-14. For each of the moves $A_{\text {IIIa }}, \ldots, A_{I I I h}$ for a knot, there are two different possibilities according to the structure of the diagram outside the local part given in the 


$$
\uparrow \uparrow \underbrace{\uparrow}_{\downarrow}
$$

Fig. 9. Oriented Reidemeister moves of type II.

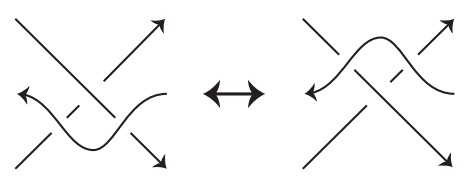

$\mathrm{A}_{\text {IIIa }}$

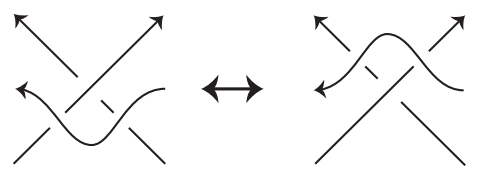

AIIIc

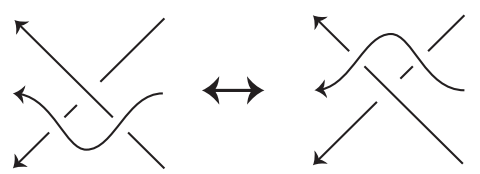

AIIIe

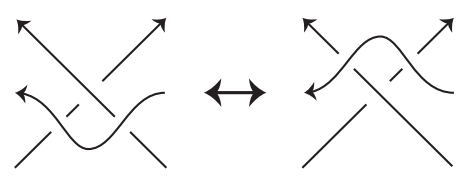

$\mathrm{A}_{\text {IIIg }}$

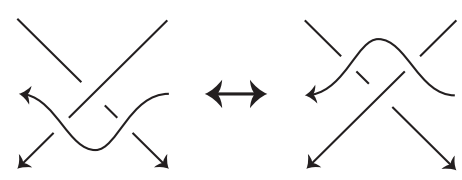

$\mathrm{A}_{\text {IIIb }}$

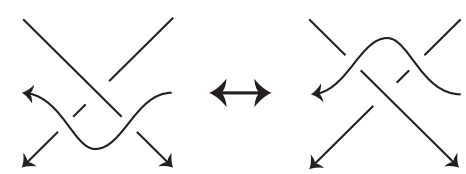

$\mathrm{A}_{\text {IIId }}$

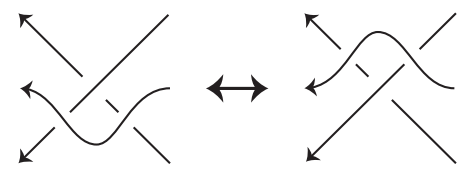

$\mathrm{A}_{\text {IIIf }}$

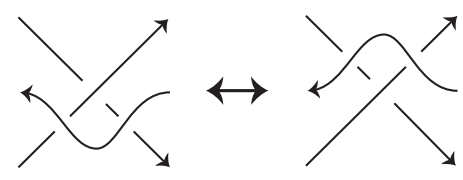

$\mathrm{A}_{\text {IIIh }}$

Fig. 10. Oriented Reidemeister moves of type III. 
diagrams in Fig. 10. By abuse of notation, we denote these moves by the same notation; for the moves $\mathrm{A}_{I I I a}, \ldots, \mathrm{A}_{I I I h}$, we denote the corresponding moves by $\mathrm{A}_{\mathrm{III}}$, $\mathrm{A}_{I I I a^{\prime}}, \ldots, \mathrm{A}_{I I I h}, \mathrm{~A}_{I I I h^{\prime}}$; cf. [2,17]. Therefore, an oriented virtual knot (that is, an oriented virtual knot diagram modulo Reidemeister moves $A_{I a}, A_{I b}, A_{I c}, A_{I d}, A_{I I a}$, $\mathrm{A}_{\text {IIb }}, \mathrm{A}_{\text {IIc }}, \mathrm{A}_{\text {IId }}, \mathrm{A}_{\text {IIIa }}, \ldots, \mathrm{A}_{\text {IIIh }}$, and virtual Reidemeister moves $\mathrm{B}_{\mathrm{I}}, \mathrm{B}_{\mathrm{II}}, \mathrm{B}_{\mathrm{III}}, \mathrm{C}$ ) is equivalent to the corresponding circular Gauss diagram considered up to moves given in Figs. 11-14.

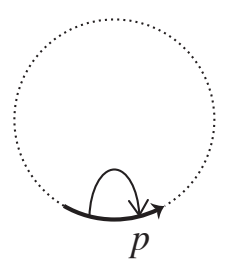

$\mathrm{A}_{\text {Ia }}(p=+)$ or $\mathrm{A}_{\text {Id }}(p=-)$

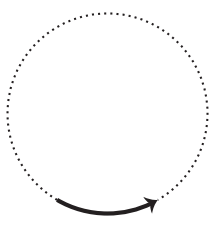

$\mathrm{A}_{\mathrm{Ib}}(p=+)$ or $\mathrm{A}_{\mathrm{Ic}}(p=-)$

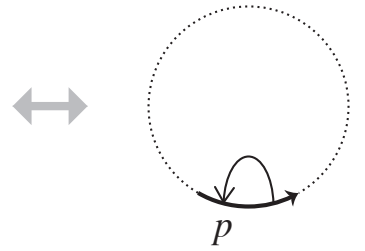

Fig. 11. Moves of circular Gauss diagrams corresponding to the oriented Reidemeister moves of type I.

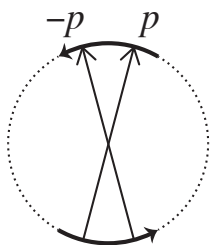

$\mathrm{A}_{\text {IIa }}(p=+)$ or $\mathrm{A}_{\text {IIb }}(p=-)$

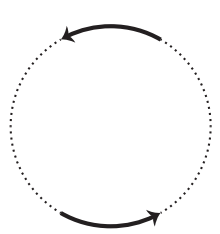

$\mathrm{A}_{\text {IIc }}(p=+)$ or $\mathrm{A}_{\text {IId }}(p=-)$

Fig. 12. Moves of circular Gauss diagrams corresponding to the oriented Reidemeister moves of type II.
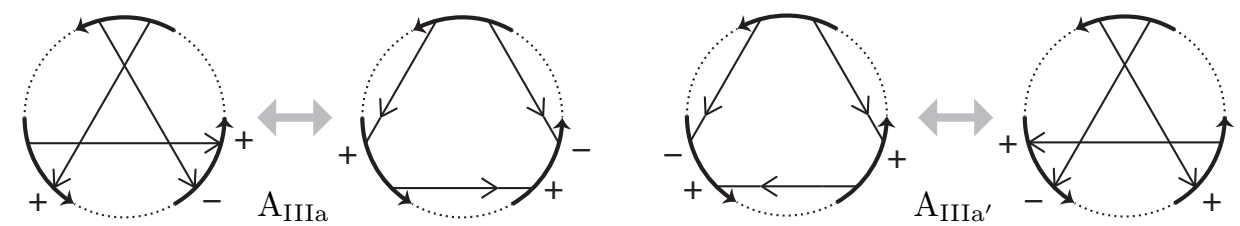

Fig. 13. Moves of circular Gauss diagrams corresponding to the oriented Reidemeister moves of type III. 

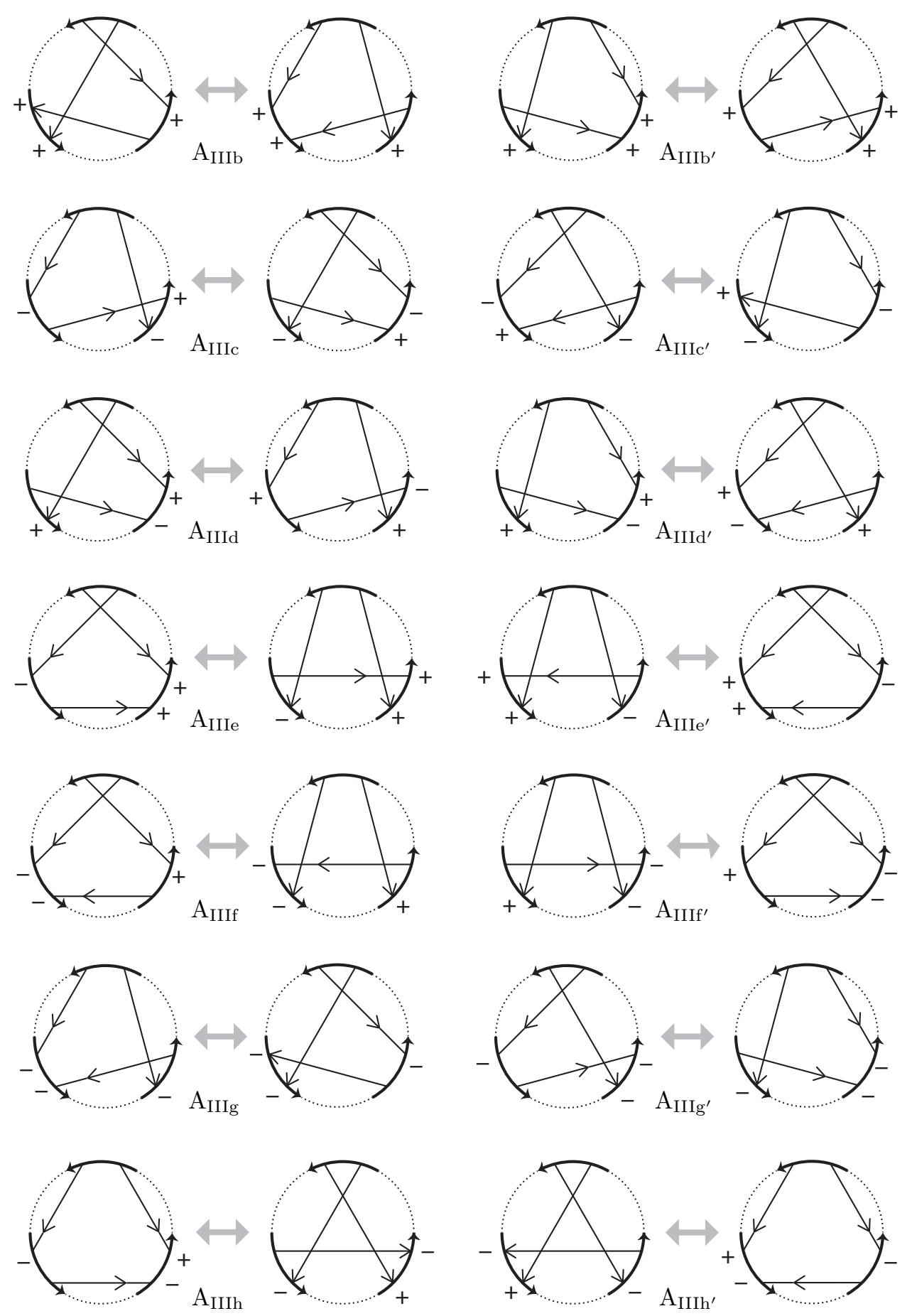

Fig. 14. Moves of circular Gauss diagrams corresponding to the oriented Reidemeister moves of type III. 
Now we consider a circular Gauss diagram for a welded knot. Depending on orientations of strings, there are four different versions of the welded move $\mathrm{D}$ as shown in Fig. 15, where $p, q= \pm$.

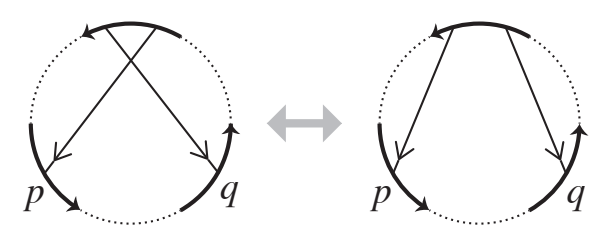

Fig. 15. Moves of circular Gauss diagrams corresponding to the move D, where $p, q= \pm$.

Therefore, if we allow the move $\mathrm{D}$, then the moves $\mathrm{A}_{\text {IIa }}$ and $\mathrm{A}_{\text {IIc }}$ are equivalent, and the moves $A_{I I b}$ and $A_{I I d}$ are equivalent. Also, the moves $A_{I I I a}, A_{I I I a}, \ldots, A_{I I I h}$, $\mathrm{A}_{\text {IIIh' }}$ are put together into the moves $\mathrm{A}_{\text {IIIx }}, \mathrm{A}_{\mathrm{IIIy}}, \mathrm{A}_{\mathrm{IIIz}}, \mathrm{A}_{\mathrm{IIIw}}$ as shown in Fig. 16, where $p, q= \pm$. We give the equivalent pairs in Table 1 , where, for example, it shows the move $\mathrm{A}_{\text {IIIa }}$ is equivalent to the move $\mathrm{A}_{\text {IVa }}$ with $(p, q)=(+,+)$. Thus, an oriented welded knot (that is, an oriented virtual knot diagram modulo Reidemeister moves, virtual Reidemeister moves, and welded move D) is equivalent to the corresponding circular Gauss diagram considered up to moves given in Figs. 11, 12, 15, and 16.

Table 1. Equivalent moves of circular Gauss diagrams allowing the move D.

\begin{tabular}{|c|c|c|c|c|}
\hline$\overline{(p, q)}$ & $(+,+)$ & $(+,-)$ & $(-,+)$ & $(-,-)$ \\
\hline $\mathrm{A}_{\mathrm{IVa}}$ & $\mathrm{A}_{I I I a}$ & $\mathrm{~A}_{\text {IIIf }}$ & $\mathrm{A}_{\mathrm{IIIe}}$ & $\mathrm{A}_{\text {IIIh }}$ \\
\hline$A_{I V b}$ & $\mathrm{~A}_{\mathrm{IIIe}^{\prime}}$ & $\mathrm{A}_{\mathrm{IIIh}}$ & $\mathrm{A}_{\mathrm{IIIa}}$ & $\mathrm{A}_{\text {IIIf }}$ \\
\hline $\mathrm{A}_{\mathrm{IVc}}$ & $\mathrm{A}_{\mathrm{IIIb}}$ & $\mathrm{A}_{\text {IIId }}$ & $\mathrm{A}_{\text {IIIc }}$ & $\mathrm{A}_{\text {IIIg' }}$ \\
\hline $\mathrm{A}_{\text {IVd }}$ & $\mathrm{A}_{\mathrm{IIIb}}$ & $\mathrm{A}_{\text {IIId }}$ & $\mathrm{A}_{\text {IIIc }}$ & $\mathrm{A}_{\text {IIIg }}$ \\
\hline
\end{tabular}

\subsection{Linear Gauss diagram}

We can define the Gauss diagram for an oriented virtual arc in a similar way to a circular Gauss diagram, which we call a linear Gauss diagram; see Fig. 17. Similarly, a linear Gauss diagram is considered up to orientation preserving homeomorphism of the underlying oriented interval. Then a linear Gauss diagram determines a virtual arc diagram, that is, if two virtual arc diagrams have the same linear Gauss diagram, then there exist a finite sequence of virtual Reidemeister moves $B_{I}, B_{I I}$, $\mathrm{B}_{\text {III }}, \mathrm{C}, \mathrm{E}_{\mathrm{I}}$ which transforms one into the other. Therefore, since a welded arc determines a ribbon 2-knot [19, Proposition 5.2], we have: 

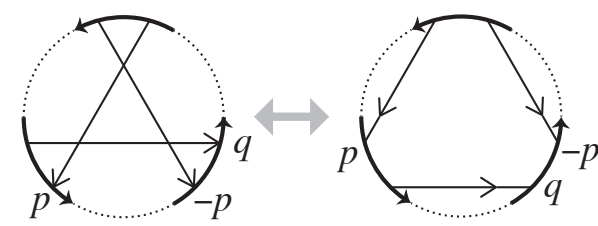

$\mathrm{A}_{\text {IIIx }}$

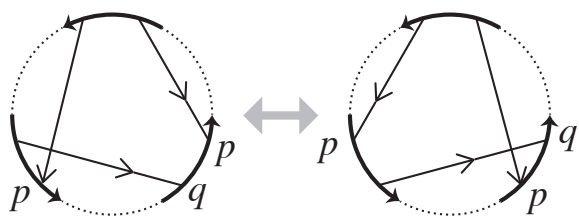

$\mathrm{A}_{\mathrm{IIIz}}$
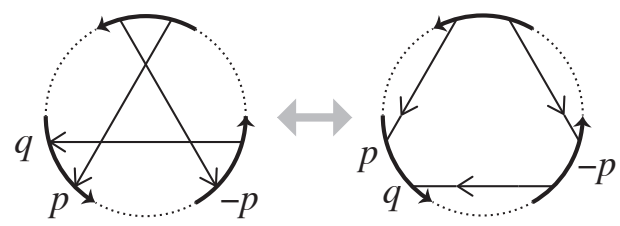

$\mathrm{A}_{\text {IIIy }}$
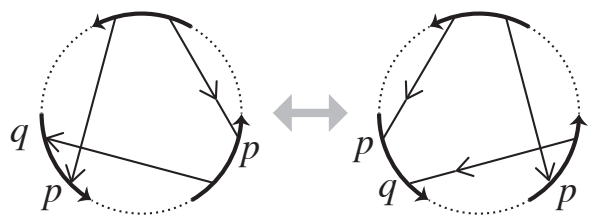

$\mathrm{A}_{\text {IIIw }}$

Fig. 16. Moves of circular Gauss diagrams equivalent to the moves $A_{I I I a}, \ldots, A_{I I I h^{\prime}}$ allowing the move $\mathrm{D}$, where $p, q= \pm$.

Proposition 3.1. A linear Gauss diagram determines an associated ribbon 2-knot.
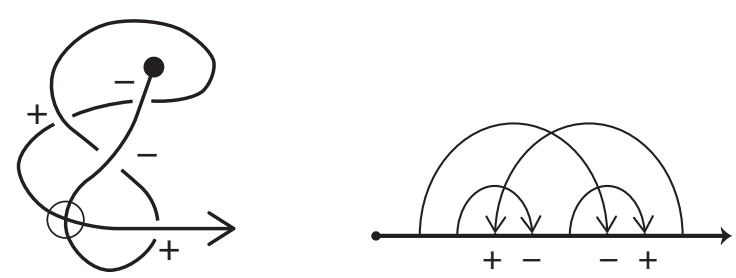

Fig. 17. Virtual arc diagram $A$ in Fig. 6 and its linear Gauss diagram $\gamma$.

By abuse of notation, we denote by $\operatorname{Tube}(\gamma)$ the ribbon 2-knot obtained from a virtual arc diagram with Gauss diagram $\gamma$. Also, we define two Gauss diagrams $\gamma$ and $\gamma^{\prime}$ are $r$-equivalent, denoted by $\gamma \stackrel{\mathrm{r}}{\sim} \gamma^{\prime}$, if they correspond to the isotopic ribbon 2-knots; $\operatorname{Tube}(\gamma) \approx \operatorname{Tube}\left(\gamma^{\prime}\right)$.

We define the moves $A_{I}, A_{I I}, A_{I I I}, D$, and $E_{I I}$ for a linear Gauss diagrams as follows:

$\mathrm{A}_{\mathrm{I}}$ : One of the 2 types of moves as shown in Fig. 18.

$\mathrm{A}_{\mathrm{II}}$ : One of the 4 types of moves as shown in Fig. 19.

$\mathrm{A}_{\mathrm{III}}$ : One of the 12 types of moves as shown in Fig. 20.

D: One of the 3 types of moves as shown in Fig. 21.

$\mathrm{E}_{\mathrm{II}}$ : One of the 2 types of moves as shown in Fig. 22. 

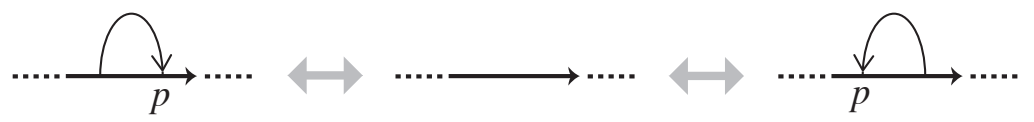

Fig. 18. Moves $\mathrm{A}_{\mathrm{I}}$ for a linear Gauss diagram, where $p= \pm$.
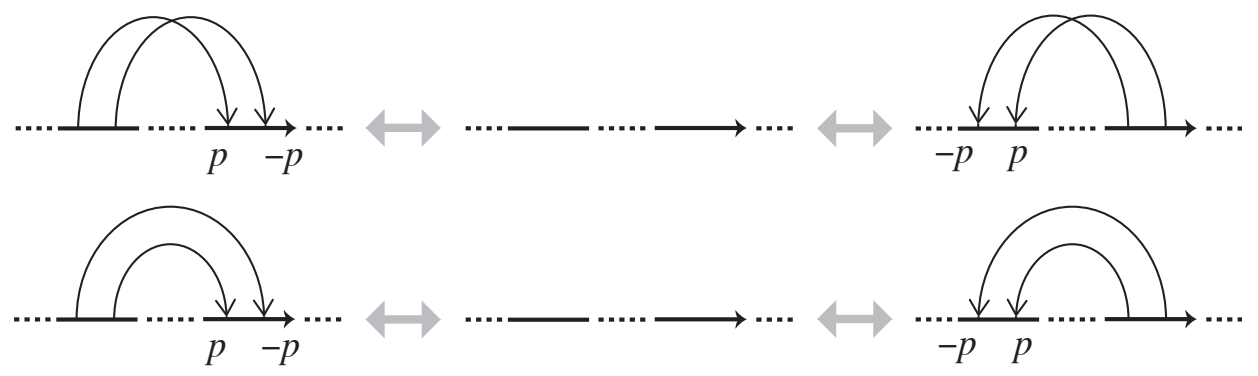

Fig. 19. Moves $\mathrm{A}_{\mathrm{II}}$ for a linear Gauss diagram, where $p= \pm$.
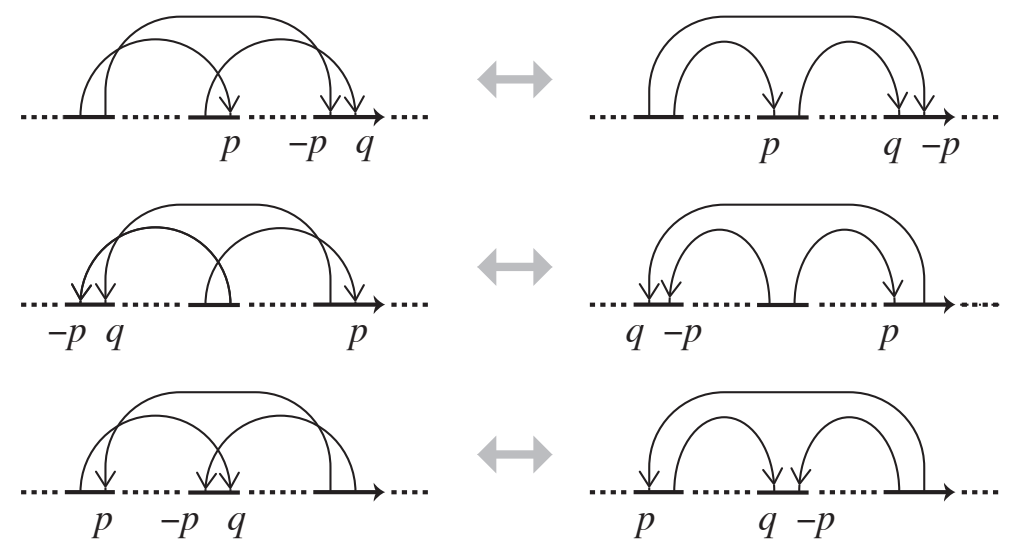

Fig. 20. Moves $\mathrm{A}_{\text {III }}$ for a linear Gauss diagram, where $p, q= \pm$.

The moves $A_{I}, A_{I I}, A_{I I I}$, and $D$ are yielded from those of a circular Gauss diagram given in Figs. 11, 12, 16, and 15, respectively, and the move $\mathrm{E}_{\mathrm{II}}$ corresponds to the welded move $\mathrm{E}_{\mathrm{II}}$ as shown in Fig. 4. So, we have:

Proposition 3.2. If two linear Gauss diagrams are related by the moves $\mathrm{A}_{\mathrm{I}}, \mathrm{A}_{\mathrm{II}}$, $\mathrm{A}_{\mathrm{III}}, \mathrm{D}$, or $\mathrm{E}_{\mathrm{II}}$, then they are r-equivalent.

Furthermore, we define the move $\Omega_{i}, i=1,2, \ldots, 8$, for a linear Gauss diagram as shown in Fig. 23. Then we have: 

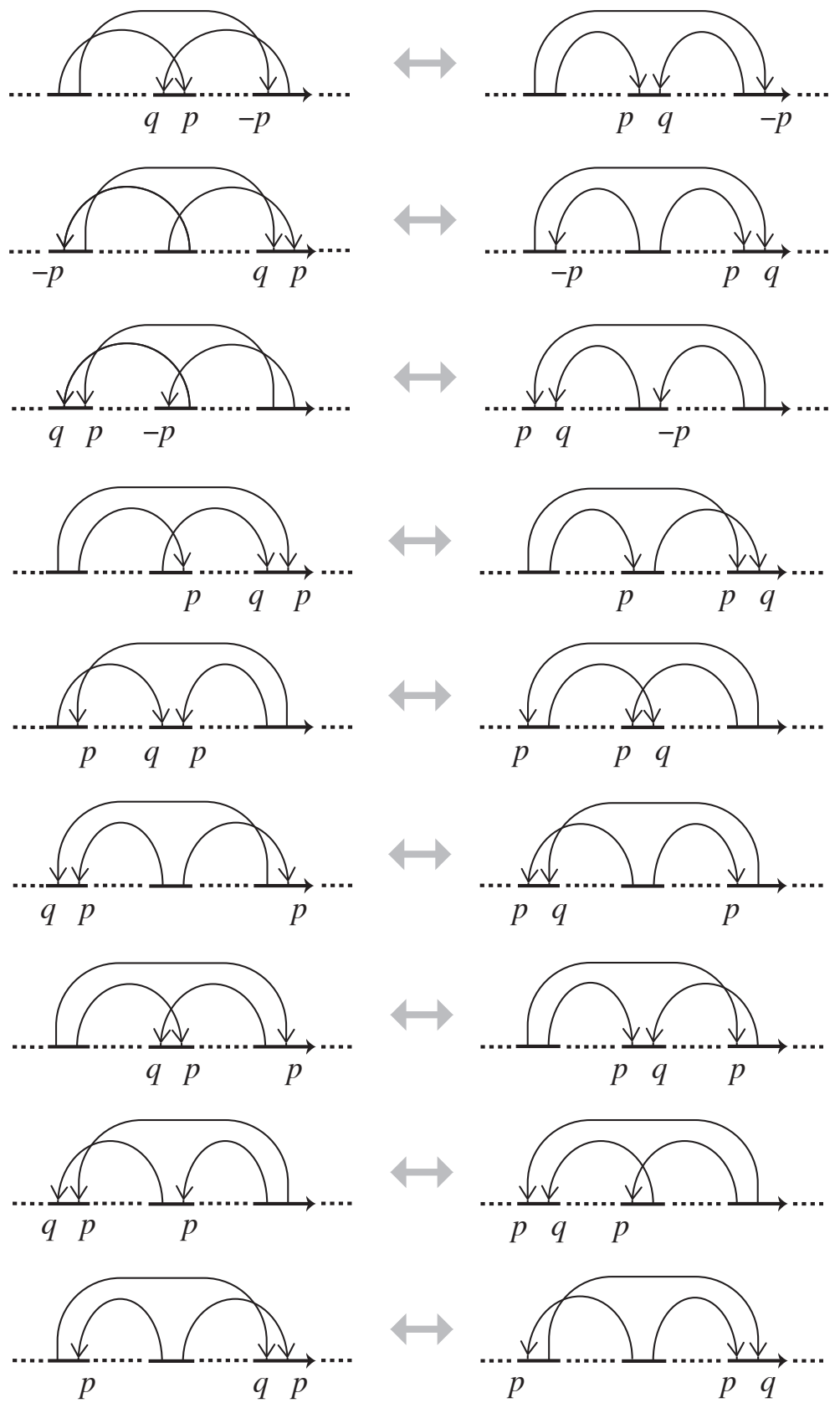

Fig. 20. Cont'd.

Proposition 3.3. If two linear Gauss diagrams are related by the move $\Omega_{i}, i=$ $1, \ldots, 8$, then they are r-equivalent. 

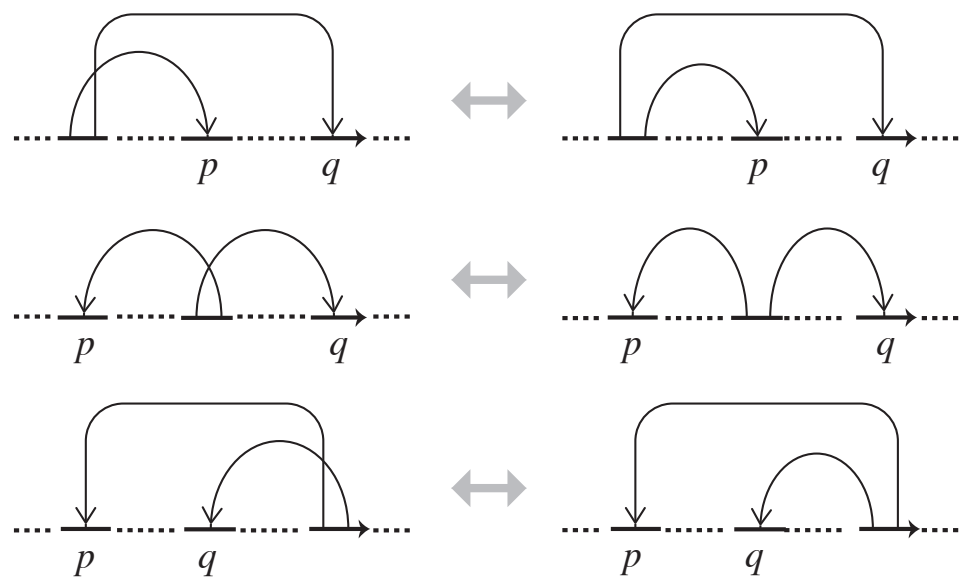

Fig. 21. Moves D for a linear Gauss diagram, where $p, q= \pm$.
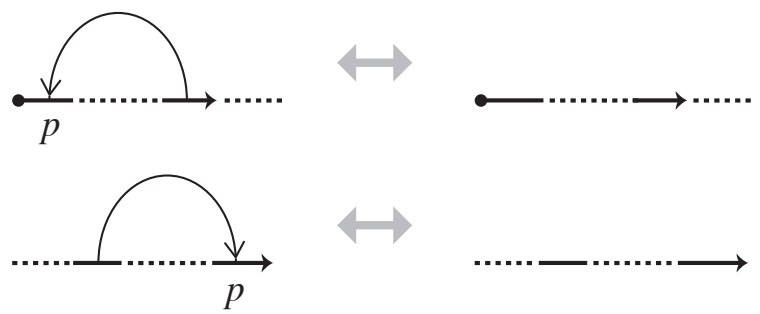

Fig. 22. Moves $\mathrm{E}_{\mathrm{II}}$ for a linear Gauss diagram, where $p= \pm$.

Proof. Each of the moves $\Omega_{1}, \Omega_{2}, \Omega_{3}, \Omega_{4}$ is realized by a sequence of the moves $\mathrm{A}_{\mathrm{I}}$ and $\mathrm{A}_{\mathrm{III}}$; for the move $\Omega_{1}$ see Fig. 24 .

Next, the move $\Omega_{1}$ implies the move $\Omega_{5}$. In fact, the $\Omega_{5}$ is realized by a sequence of the moves $\mathrm{D}$ and $\mathrm{E}_{\mathrm{II}}$ as shown in Fig. 25. Similarly, the moves $\Omega_{1}, \Omega_{3}, \Omega_{4}$ imply the moves $\Omega_{6}, \Omega_{7}, \Omega_{8}$, respectively. This completes the proof.

Let $\gamma$ be the Gauss diagram of a virtual arc $A$. We denote by $\gamma^{\dagger}$ and $-\gamma$ the Gauss diagrams obtained from $\gamma$ by changing the signs of chords and by reversing the orientation of the underlying interval, respectively. Then $\gamma^{\dagger}$ and $-\gamma$ are the Gauss diagrams of the virtual arcs $A^{\dagger}$ and $-A$, respectively. In particular, the Gauss diagram $-\gamma^{\dagger}$ is obtained from $\gamma$ by changing the signs of chords and reversing the orientation of the interval, which is the Gauss diagrams of the virtual arc $-A^{\dagger}$.

Definition 3.4. We call the change of a linear Gauss diagram $\gamma \mapsto-\gamma^{\dagger}$ the $r e$ versing move; see Fig. 26.

Then by Proposition 2.2, we have 

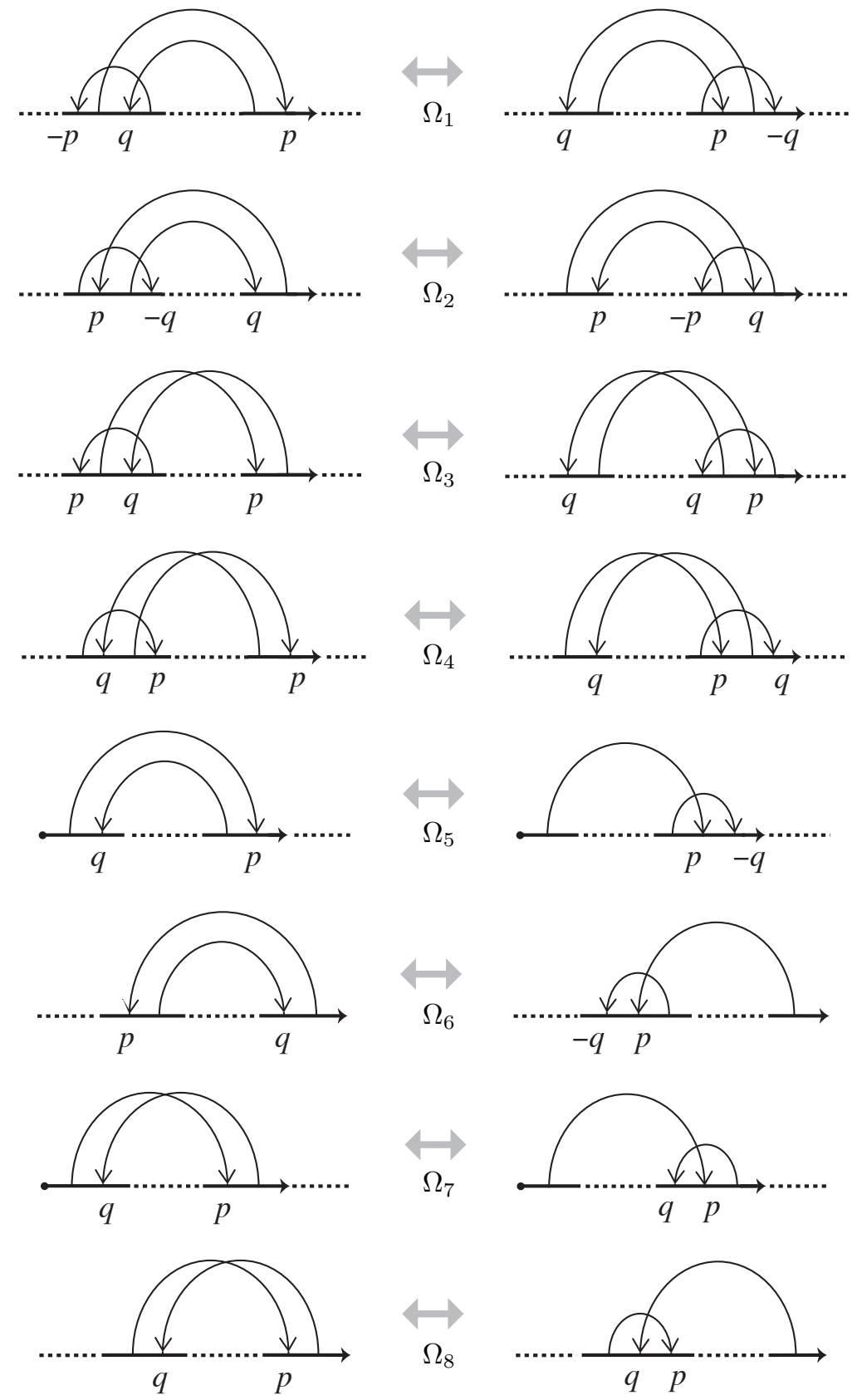

Fig. 23. Moves $\Omega_{i}, i=1, \ldots, 8$, which preserve the r-equivalence. 

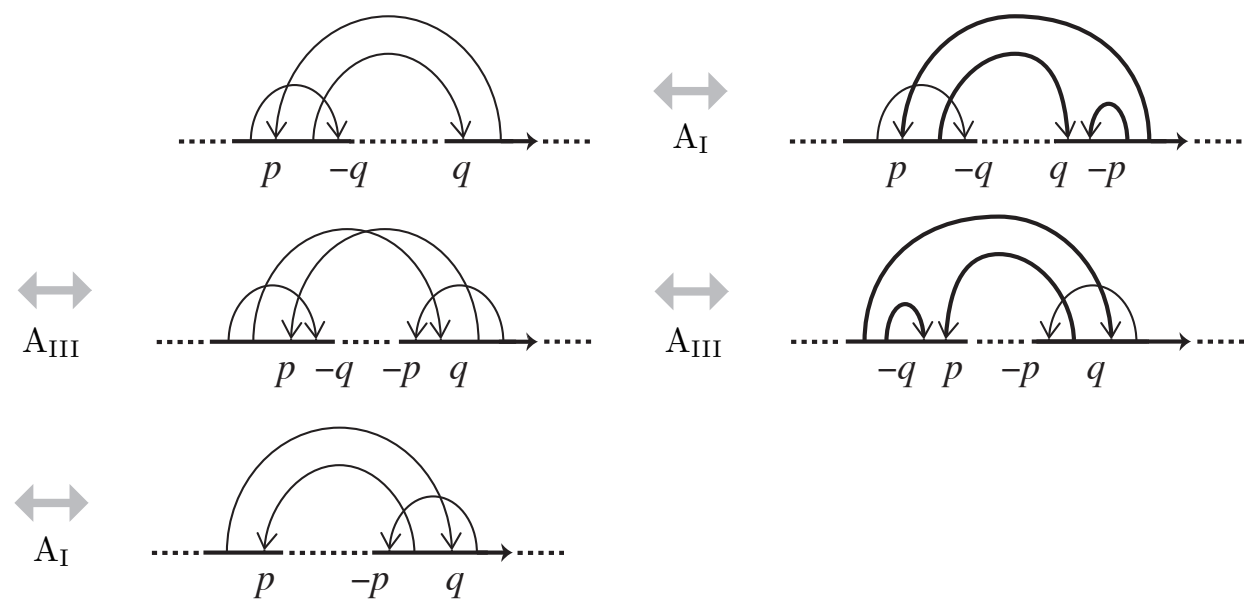

Fig. 24. Proof of Proposition 3.3 for the move $\Omega_{1}$.
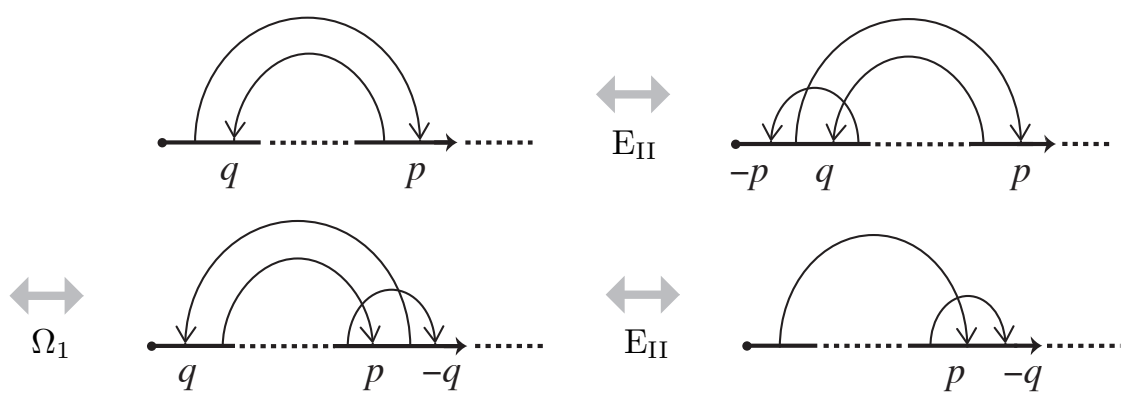

Fig. 25. Proof of Proposition 3.3 for the move $\Omega_{5}$.
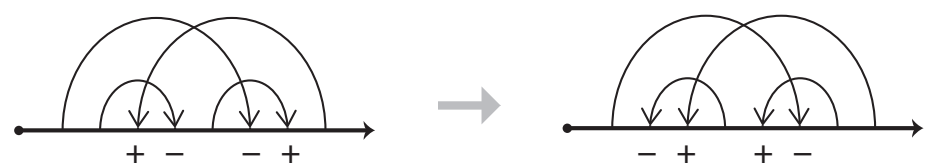

Fig. 26. Reversion move $\gamma \mapsto-\gamma^{\dagger}$, where $\gamma$ and $-\gamma^{\dagger}$ are the linear Gauss diagrams for the virtual $\operatorname{arcs} A$ and $-A^{\dagger}$ given in Fig. 6 .

Proposition 3.5. The reversing move of a linear Gauss diagram preserves the r-equivalence, $\gamma \stackrel{\mathrm{r}}{\sim}-\gamma^{\dagger}$.

Let $\gamma$ and $\gamma^{\prime}$ be the Gauss diagrams of virtual arcs $A$ and $A^{\prime}$. We define the product $\gamma \cdot \gamma^{\prime}$ by connecting the terminal point of the underlying interval of $\gamma$ to the initial point of the underlying interval of $\gamma^{\prime}$; see Fig. 27. Then $\gamma \cdot \gamma^{\prime}$ is the Gauss diagram of the product virtual arc $A \cdot A^{\prime}$. 
The associated ribbon 2-knots Tube $\left(\gamma \cdot \gamma^{\prime}\right)$ represents the composition of Tube $(\gamma)$ and Tube $\left(\gamma^{\prime}\right)$. Since the composition of oriented 2-knots is well defined up to isotopy, we have the following:

$$
\gamma \cdot \gamma^{\prime} \stackrel{\mathrm{r}}{\sim} \gamma^{\prime} \cdot \gamma \stackrel{\mathrm{r}}{\sim}\left(-\gamma^{\dagger}\right) \cdot \gamma^{\prime}
$$

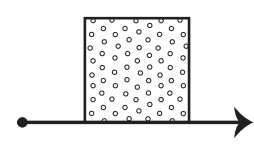

$\gamma$

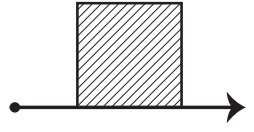

$\gamma^{\prime}$
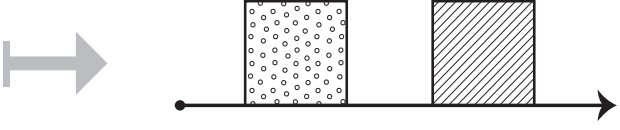

$\gamma \cdot \gamma^{\prime}$

Fig. 27. Product of Gauss diagrams.

By Proposition 2.2 two ribbon 2-knots Tube $\left(\gamma^{\dagger}\right)$ and Tube $(-\gamma)$ are isotopic, which are the mirror images of $\operatorname{Tube}(\gamma)$, and thus, we have

$$
\gamma \stackrel{\mathrm{r}}{\sim}-\gamma^{\dagger}
$$

If a virtual arc diagram $A$ has a nugatory crossing as in Fig. 28(a), then $A$ may be deformed into $A^{\prime}$ as in Fig. 28(b) by the Reidemeister moves and welded moves. Therefore, we have the following:

Lemma 3.6. If a Gauss diagram $\gamma$ has a separated single chord $c$ as in Fig. 28(c) with any orientation and sign, then it is r-equivalent to the Gauss diagram obtained from $\gamma$ by deleting the chord $c$.

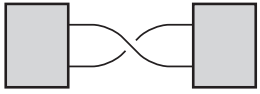

(a)

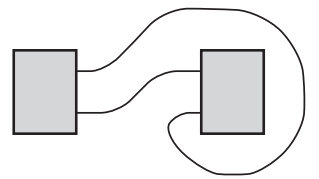

(b)

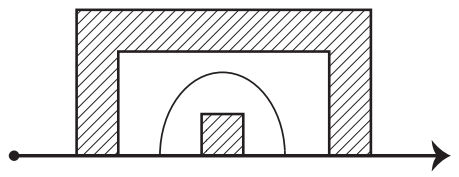

(c)

Fig. 28. (a) Virtual arc diagram $A$ with a nugatory crossing; (b) virtual arc diagram $A^{\prime}$; (c) Gauss diagram with a separated single chord.

\section{Enumeration Result}

Before stating the main result, we give some convention of a linear Gauss diagram which presents a ribbon 2-knot. Given a linear Gauss diagram we order its chords following the orientation of the underlying interval. More precisely, let $\gamma$ be a linear 
Gauss diagram with $n$ chords such that $2 n$ endpoints lie in the underlying interval $[0,1]$, where 0 is the initial point and 1 is the terminal point. We order the chords $c_{1}, c_{2}, \ldots, c_{n}$ of $\gamma$ so that $h\left(c_{i}\right)<h\left(c_{i+1}\right)$, where $h\left(c_{i}\right)$ is the arrowhead point of $c_{i}$. If the chord $c_{i}$ has the sign $p_{i}, p_{i}= \pm$, then we denote $\gamma$ by $\gamma\left(p_{1}, p_{2}, \ldots, p_{n}\right)$.

The following is our main result.

Theorem 4.1. A ribbon 2-knot presented by a virtual arc with up to 4 crossings is either the trivial one or one of those listed in Tables 2, 3 and 4.

Each column in Tables 2, 3 and 4 shows as follows:

- The first column, Name, shows the name of the corresponding ribbon 2-knot, where $K$ ! denotes the mirror image of the knot $K$, and $K \# K^{\prime}$ the composition of two knots $K$ and $K^{\prime}$.

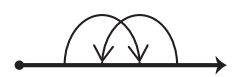

$\Gamma_{1}^{2}$

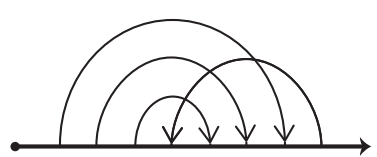

$\Gamma_{1}^{4}$

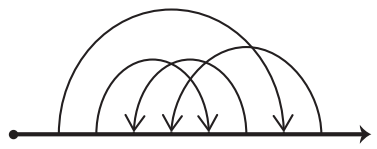

$\Gamma_{4}^{4}$

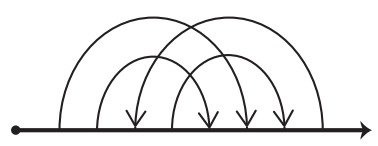

$\Gamma_{7}^{4}$

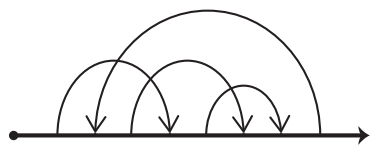

$\Gamma_{10}^{4}$

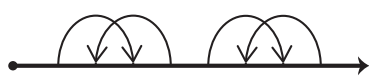

$\Gamma_{13}^{4}$

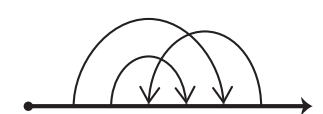

$\Gamma_{1}^{3}$

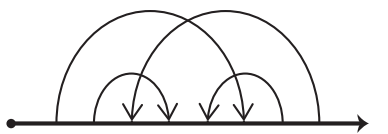

$\Gamma_{2}^{4}$

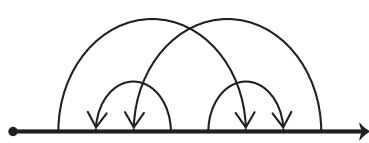

$\Gamma_{5}^{4}$

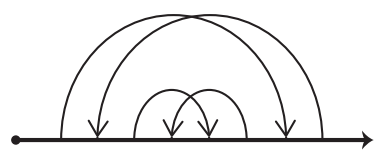

$\Gamma_{8}^{4}$

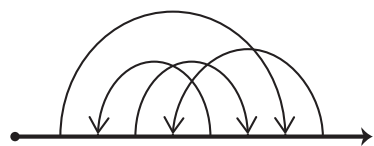

$\Gamma_{11}^{4}$

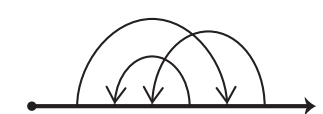

$\Gamma_{2}^{3}$

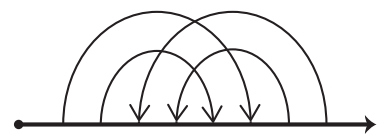

$\Gamma_{3}^{4}$

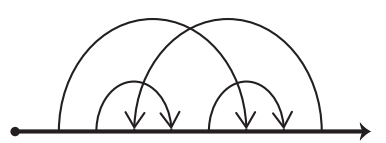

$\Gamma_{6}^{4}$

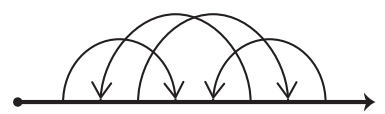

$\Gamma_{9}^{4}$

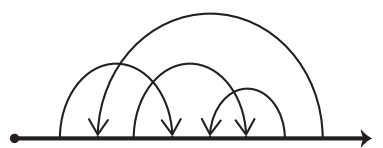

$\Gamma_{12}^{4}$

Fig. 29. Linear Gauss diagrams with up to 4 chords. 
- The second column, Gauss diagram, shows the linear Gauss diagram presenting the ribbon 2-knot, which are shown as in Fig. 29. If there are more than one diagram, then they present isotopic ribbon 2-knots.

- The column, Group, in Tables 2 and 3 shows the group presentation of the ribbon 2-knot, that is, the fundamental group of the complement of the ribbon 2-knot, where

$$
G\left(a_{1}, b_{1}, \ldots, a_{n}, b_{n}\right)=\left\langle x, y ; y=w x w^{-1}\right\rangle, \quad w=x^{a_{1}} y^{b_{1}} \cdots x^{a_{n}} y^{b_{n}} .
$$

Note that $x, y$ are meridians. In Table 4 there is no column listing the knot group because those ribbon 2-knots are composite and do not have such a group presentation.

- The column, $\Delta(t)$, shows the Alexander polynomial of the ribbon 2-knot which is normalized so that $\Delta(1)=1$ and $(d / d t) \Delta(1)=0$. We abbreviate $\Delta(t)$ as follows: for $c_{i} \in Z$

$$
\left(\begin{array}{lllllllll}
c_{-m} & c_{-m+1} & \cdots & c_{-1} & {\left[c_{0}\right]} & c_{1} & c_{2} & \cdots & c_{n}
\end{array}\right)=\sum_{i=-m}^{n} c_{i} t^{i} .
$$

- The column, Det, shows the determinant of the ribbon 2-knot which is given by $|\Delta(-1)|$.

- In the last column if there is a mark "a", then the ribbon 2-knot is amphicheiral.

Remark 4.2. The group $G\left(a_{1}, b_{1}, \ldots, a_{n}, b_{n}\right)$ is isomorphic to $G\left(-b_{n},-a_{n}, \ldots,-b_{1},-a_{1}\right)$. In fact, $\left\langle x, y ; y=w x w^{-1}\right\rangle=\left\langle x, y ; x=w^{-1} y w\right\rangle$, where $w^{-1}=y^{-b_{n}} x^{-a_{n}} \cdots y^{-b_{1}} x^{-a_{1}}$ for $w=x^{a_{1}} y^{b_{1}} \cdots x^{a_{n}} y^{b_{n}}$. This is realized by the reversing move of a linear Gauss diagram.

Table 2. Ribbon 2-knots presented by linear Gauss diagrams with 2 or 3

\begin{tabular}{|c|c|c|c|c|c|}
\hline Name & Gauss diagram & Group & $\Delta(t)$ & Det & \\
\hline$\overline{R_{1}^{2}}$ & $\Gamma_{1}^{2}(++), \Gamma_{1}^{2}(--)$ & $G(1,1)$ & $(1[-1] 1)$ & 3 & $\mathrm{a}$ \\
\hline$R_{2}^{2}$ & $\Gamma_{1}^{2}(+-)$ & $G(1,-1)$ & $([0] 2-1)$ & 3 & \\
\hline$R_{2}^{2} !$ & $\Gamma_{1}^{2}(-+)$ & $G(-1,1)$ & $\left(\begin{array}{lll}-1 & 2 & {[0]}\end{array}\right)$ & 3 & \\
\hline$R_{1}^{3}$ & $\Gamma_{1}^{3}(+++)$ & $G(1,2)$ & $\left(\begin{array}{llll}1 & -1 & {[0]} & 1\end{array}\right)$ & 1 & \\
\hline$R_{1}^{3} !$ & $\Gamma_{1}^{3}(---)$ & $G(-1,-2)$ & $\left(\begin{array}{llll}1 & {[0]} & -1 & 1\end{array}\right)$ & 1 & \\
\hline$R_{2}^{3}$ & $\Gamma_{1}^{3}(+--)$ & $G(1,-2)$ & $\left([0]\left[\begin{array}{lll}{[} & 1 & -1\end{array}\right)\right.$ & 1 & \\
\hline$R_{2}^{3}$ ! & $\Gamma_{1}^{\frac{1}{3}}(-++)$ & $G(-1,2)$ & $\left(\begin{array}{llll}-1 & 1 & 1 & {[0]}\end{array}\right)$ & 1 & \\
\hline$R_{3}^{3}$ & $\Gamma_{2}^{3}(+++), \Gamma_{2}^{3}(+--)$ & $G(1,-1,1,1)$ & $\left(\left[\begin{array}{lll}2 & -2 & 1\end{array}\right)\right.$ & 5 & \\
\hline$R_{3}^{3}$ ! & $\Gamma_{2}^{3}(---), \Gamma_{2}^{3}(-++)$ & $G(-1,1,-1,-1)$ & $(1-2[2])$ & 5 & \\
\hline$R_{4}^{3}$ & $\Gamma_{2}^{3}(++-)$ & $G(1,1,1,-1)$ & $([1]-1 \quad 2-1)$ & 5 & \\
\hline$R_{4}^{3} !$ & $\Gamma_{2}^{3}(--+)$ & $G(-1,-1,-1,1)$ & $\left(\begin{array}{lll}-1 & 2 & -1\end{array}[1]\right)$ & 5 & \\
\hline$R_{5}^{3}$ & $\Gamma_{2}^{3}(+-+), \Gamma_{2}^{3}(-+-)$ & $G(1,-1,-1,1)$ & $(-1[3]-1)$ & 5 & $\mathrm{a}$ \\
\hline
\end{tabular}
chords. 
Table 3. Ribbon 2-knots presented by the linear Gauss diagrams $\Gamma_{l}^{4}, 1 \leq l \leq 12$.

\begin{tabular}{|c|c|c|c|c|c|}
\hline Name & Gauss diagram & Group & $\Delta(t)$ & Det & \\
\hline$R_{1,1}^{4}$ & $\Gamma_{1}^{4}(++++)$ & $G(1,3)$ & $\left(\begin{array}{lllll}1 & -1 & 0 & {[0]} & 1\end{array}\right)$ & 3 & \\
\hline$R_{1,1}^{4} !$ & $\Gamma_{1}^{4}(----)$ & $G(-1,-3)$ & $\left(\begin{array}{lllll}1 & {[0]} & 0 & -1 & 1\end{array}\right)$ & 3 & \\
\hline$R_{1,2}^{4}$ & $\Gamma_{1}^{4}(-+++)$ & $G(-1,3)$ & $\left(\begin{array}{lllll}-1 & 1 & 0 & 1 & {[0]}\end{array}\right)$ & 3 & \\
\hline$R_{1,2}^{4} !$ & $\Gamma_{1}^{4}(+---)$ & $G(1,-3)$ & 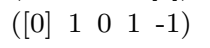 & 3 & \\
\hline$R_{2,1}^{4}$ & $\Gamma_{2}^{4}(++++), \Gamma_{2}^{4}(----)$ & $G(1,1,1,1)$ & $\left.\left(\begin{array}{lllll}1 & -1 & {[1}\end{array}\right]-1 \quad 1\right)$ & 5 & a \\
\hline$R_{2,2}^{4,1}$ & $\Gamma_{2}^{4}(+-+-)$ & $G(1,-1,1,-1)$ & $([0] \quad 003-2)$ & 5 & \\
\hline$R_{2,2}^{4,2} !$ & $\Gamma_{2}^{4}(-+-+)$ & $G(-1,1,-1,1)$ & $\left(\begin{array}{llll}-2 & 3 & 0 & {[0]}\end{array}\right)$ & 5 & \\
\hline$R_{3,1}^{4}$ & $\Gamma_{3}^{4}(++++), \Gamma_{3}^{4}(----)$ & $G(2,2)$ & $\left(\begin{array}{lllll}1 & 0 & {[-1]} & 0 & 1\end{array}\right)$ & 1 & $\mathrm{a}$ \\
\hline$R_{3,2}^{4}$ & $\Gamma_{3}^{4}(++--)$ & $G(2,-2)$ & $([0] \quad 002000-1)$ & 1 & \\
\hline$R_{3,2}^{4} !$ & $\Gamma_{3}^{4}(--++)$ & $G(-2,2)$ & $\left(\begin{array}{lllll}-1 & 0 & 2 & 0 & [0])\end{array}\right)$ & 1 & \\
\hline$R_{4,1}^{4}$ & $\Gamma_{4}^{4}(++++)$ & $G(1,-1,1,2)$ & $\left(\begin{array}{llll}2 & {[-2]} & 0 & 1\end{array}\right)$ & 3 & \\
\hline$R_{4,1}^{4,1} !$ & $\Gamma_{4}^{4}(----)$ & $G(-1,1,-1,-2)$ & $\left(\begin{array}{llll}1 & 0 & {[-2} & 2\end{array}\right)$ & 3 & \\
\hline$R_{4,2}^{4}$ & $\Gamma_{4}^{4}(+-++)$ & $G(1,-1,-1,2)$ & $\left(\begin{array}{lll}-1 & 2 & {[0]}\end{array}\right)$ & 3 & \\
\hline$R_{4,2}^{4} !$ & $\Gamma_{4}^{4}(-+--)$ & $G(-1,1,1,-2)$ & $([0] 2-1)$ & 3 & \\
\hline$R_{4,3}^{4}$ & $\Gamma_{4}^{4}(-+++)$ & $G(-1,-1,1,2)$ & $\left(\begin{array}{llll}1 & -2 & 1 & {[1]}\end{array}\right)$ & 3 & \\
\hline$R_{4,3}^{4} !$ & $\Gamma_{4}^{4}(+---)$ & $G(1,1,-1,-2)$ & $\left(\left[\begin{array}{llll}1 & 1 & -2 & 1\end{array}\right)\right.$ & 3 & \\
\hline$R_{4,4}^{4}$ & $\Gamma_{4}^{4}(++--)$ & $G(1,1,1,2)$ & 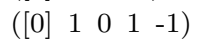 & 3 & \\
\hline$R_{4,4}^{4} !$ & $\Gamma_{4}^{4}(--++)$ & $G(-1,-1,-1,-2)$ & $\left(\begin{array}{lllll}-1 & 1 & 0 & 1 & {[0]}\end{array}\right)$ & 3 & \\
\hline$R_{5,1}^{4}$ & $\Gamma_{5}^{4}(++--)$ & $G(1,-1,-1,-2)$ & $\left(\left[\begin{array}{lllll}1] & 0 & -1 & 2 & -1\end{array}\right)\right.$ & 3 & \\
\hline$R_{5,1}^{4,1} !$ & $\Gamma_{5}^{4}(--++)$ & $G(-1,1,1,2)$ & $\left(\begin{array}{lllll}-1 & 2 & -1 & 0 & {[1]}\end{array}\right)$ & 3 & \\
\hline$R_{6,1}^{4}$ & $\Gamma_{6}^{4}(++++)$ & $G(1,1,-1,1,1,1)$ & $\left(\begin{array}{lllll}1 & -2 & 2 & {[-1]} & 1\end{array}\right)$ & 7 & \\
\hline$R_{6,1}^{4,1} !$ & $\Gamma_{6}^{4}(----)$ & $G(-1,-1,1,-1,-1,-1)$ & $\left(\begin{array}{lllll}1 & {[-1]} & 2 & -2 & 1\end{array}\right)$ & 7 & \\
\hline$R_{6,2}^{4,1}$ & $\Gamma_{6}^{4}(+++-), \Gamma_{6}^{4}(---+)$ & $G(1,1,-1,-1,1,1)$ & $(2[-3] 2)$ & 7 & $\mathrm{a}$ \\
\hline$R_{6,3}^{4,2}$ & $\begin{array}{l}\Gamma_{6}^{4}(++-+), \Gamma_{6}^{4}(-+--), \\
\Gamma_{6}^{4}(--++)\end{array}$ & $G(1,-1,-1,1,1,1)$ & $\left(\begin{array}{llll}-1 & 3 & {[-2]} & 1\end{array}\right)$ & 7 & \\
\hline$R_{6,3}^{4} !$ & $\begin{array}{l}\Gamma_{6}^{4}(--+-), \Gamma_{6}^{4}(+-++) \\
\Gamma_{6}^{4}(++--)\end{array}$ & $G(-1,1,1,-1,-1,-1)$ & $(1[-2] 3-1)$ & 7 & \\
\hline$R_{6,4}^{4}$ & $\Gamma_{6}^{4}(-+++)$ & $G(-1,1,1,1,-1,1)$ & $\left(\begin{array}{lllll}-1 & 2 & -2 & 2 & {[0]}\end{array}\right)$ & 7 & \\
\hline$R_{6,4}^{4} !$ & $\Gamma_{6}^{4}(+---)$ & $G(1,-1,-1,-1,1,-1)$ & $([0] \quad 2 \quad-2 \quad 2 \quad-1)$ & 7 & \\
\hline$R_{6,5}^{4}$ & $\Gamma_{6}^{4}(+-+-)$ & $G(1,1,-1,-1,1,-1)$ & $\left(\left[\begin{array}{llll}0] & 3 & -3 & 1\end{array}\right)\right.$ & 7 & \\
\hline$R_{6,5}^{4} !$ & $\Gamma_{6}^{4}(-+-+)$ & $G(-1,-1,1,1,-1,1)$ & $\left(\begin{array}{llll}1 & -3 & 3 & {[0]}\end{array}\right)$ & 7 & \\
\hline$R_{6,6}^{4}$ & $\Gamma_{6}^{4}(-++-)$ & $G(-1,1,1,-1,-1,1)$ & $(-2 \quad 4[-1])$ & 7 & \\
\hline$R_{6,6}^{4} !$ & $\Gamma_{6}^{4}(+--+)$ & $G(1,-1,-1,1,1,-1)$ & $([-1] 4-2)$ & 7 & \\
\hline$R_{7,1}^{4}$ & $\Gamma_{7}^{4}(++++)$ & $G(-1,1,-2,-1)$ & $\left(\begin{array}{llll}1 & -1 & -1 & {[2]}\end{array}\right)$ & 1 & \\
\hline$R_{7,1}^{4} !$ & $\Gamma_{7}^{4}(----)$ & $G(1,-1,2,1)$ & $([2]-1 \quad-1 \quad 1)$ & 1 & \\
\hline$R_{7,2}^{4,1}$ & $\Gamma_{7}^{4}(+++-)$ & $G(1,1,-2,-1)$ & 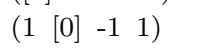 & 1 & \\
\hline$R_{7,2}^{4 ! 2} !$ & $\Gamma_{7}^{4}(---+)$ & $G(-1,-1,2,1)$ & $\left(\begin{array}{llll}1 & -1 & {[0]} & 1\end{array}\right)$ & 1 & \\
\hline$R_{7,3}^{4,2}$ & $\Gamma_{7}^{4}(-+++)$ & $G(-1,-1,-2,1)$ & $\left(\begin{array}{lllll}-1 & 1 & 1 & -1 & {[1]}\end{array}\right)$ & 1 & \\
\hline$R_{7,3}^{4,3} !$ & $\Gamma_{7}^{4}(+---)$ & $G(1,1,2,-1)$ & $\left(\left[\begin{array}{lllll}1] & -1 & 1 & 1 & -1\end{array}\right)\right.$ & 1 & \\
\hline$R_{7,4}^{4,3}$ & $\Gamma_{7}^{4}(-++-)$ & $G(1,-1,-2,1)$ & $\left(\begin{array}{llll}-1 & 1 & {[2]} & -1\end{array}\right)$ & 1 & \\
\hline$R_{7,4}^{4 !} !$ & $\Gamma_{7}^{4}(+--+)$ & $G(-1,1,2,-1)$ & $\left(\begin{array}{llll}-1 & {[2]} & 1 & -1\end{array}\right)$ & 1 & \\
\hline
\end{tabular}


Table 3. Cont'd.

\begin{tabular}{|c|c|c|c|c|c|}
\hline Name & Gauss diagram & Group & $\Delta(t)$ & Det & \\
\hline$R_{8,1}^{4}$ & $\begin{array}{l}\Gamma_{8}^{4}(++++), \\
\Gamma_{8}^{4}(----)\end{array}$ & $G(1,1,1,-1,-1,1,1,1)$ & $\left(\begin{array}{lllll}1 & -2 & {[3]} & -2 & 1\end{array}\right)$ & 9 & \\
\hline$R_{8,2}^{4}$ & $\begin{array}{l}\Gamma_{8}^{4}(+++-) \\
\Gamma_{8}^{4}(+---)\end{array}$ & $G(1,-1,1,1,-1,1,1,-1)$ & $\left(\left[\begin{array}{llll}2 & -3 & 3 & -1\end{array}\right)\right.$ & 9 & \\
\hline$R_{8,2}^{4} !$ & $\begin{array}{l}\Gamma_{8}^{4}(---+) \\
\Gamma_{8}^{4}(-+++)\end{array}$ & $G(-1,1,-1,-1,1,-1,-1,1)$ & $\left(\begin{array}{llll}-1 & 3 & -3 & {[2]}\end{array}\right)$ & 9 & \\
\hline$R_{8,4}^{4}$ & $\Gamma_{8}^{4}(++--)$ & $G(1,-1,1,-1,-1,-1,1,-1)$ & $\left(\left[\begin{array}{lllll}0 & 0 & 4 & -4 & 1\end{array}\right)\right.$ & 9 & \\
\hline$R_{8,4}^{4,4} !$ & $\Gamma_{8}^{4}(--++)$ & $G(-1,1,-1,1,1,1,-1,1)$ & $\left(\begin{array}{lllll}1 & -4 & 4 & 0 & {[0]}\end{array}\right)$ & 9 & \\
\hline$R_{8,5}^{4}$ & $\Gamma_{8}^{4}(+-+-)$ & $G(1,-1,-1,1,-1,1,1,-1)$ & $(-2[5]-2)$ & 9 & \\
\hline$R_{8,5}^{4,0} !$ & $\Gamma_{8}^{4}(-+-+)$ & $G(-1,1,1,-1,1,-1,-1,1)$ & $(-2[5]-2)$ & 9 & \\
\hline$R_{8,6}^{4}$ & $\begin{array}{l}\Gamma_{8}^{4}(-++-) \\
\Gamma_{8}^{4}(+--+)\end{array}$ & $G(-1,-1,1,1,1,1,-1,-1)$ & 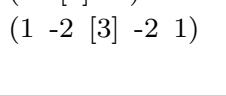 & 9 & a \\
\hline$R_{9,1}^{4}$ & $\Gamma_{9}^{4}(++--)$ & $G(1,1,-1,1,-1,-1)$ & $(2[-3] 2)$ & 7 & \\
\hline$R_{9,1}^{4,1} !$ & $\Gamma_{9}^{4}(--++)$ & $G(-1,-1,1,-1,1,1)$ & $(2[-3] 2)$ & 7 & \\
\hline$R_{9,2}^{4,1}$ & $\begin{array}{l}\Gamma_{9}^{4}(-++-) \\
\Gamma_{9}^{4}(+--+)\end{array}$ & $G(-1,1,1,1,1,-1)$ & $\left(\begin{array}{lllll}-1 & 2 & {[-1]} & 2 & -1\end{array}\right)$ & 7 & a \\
\hline$R_{10,1}^{4}$ & $\Gamma_{10}^{4}(++++)$ & $G(1,1,-1,1,1,-1,-1,1,1,1)$ & $\left(\begin{array}{lllll}1 & -3 & 4 & {[-2]} & 1\end{array}\right)$ & 11 & \\
\hline$R_{10,1}^{4} !$ & $\Gamma_{10}^{4}(----)$ & $G(-1,-1,1,-1,-1,1,1,-1,-1,-1)$ & $\left(\begin{array}{lllll}1 & {[-2]} & 4 & -3 & 1\end{array}\right)$ & 11 & \\
\hline$R_{10,2}^{4}$ & $\Gamma_{10}^{4}(+++-)$ & $G(1,1,-1,1,1,-1,-1,-1,1,1)$ & $(2[-4] 4-1)$ & 11 & \\
\hline$R_{10,2}^{10,2} !$ & $\Gamma_{10}^{4}(---+)$ & $G(-1,-1,1,-1,-1,1,1,1,1,1)$ & 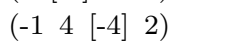 & 11 & \\
\hline$R_{10,3}^{10,2}$ & $\Gamma_{10}^{4}(++-+)$ & $G(1,1,-1,-1,1,-1,-1,1,1,1)$ & $\left(\begin{array}{llll}-1 & 4 & {[-4]} & 2\end{array}\right)$ & 11 & \\
\hline$R_{10,3}^{10,3} !$ & $\Gamma_{10}^{4}(--+-)$ & $G(-1,-1,1,1,-1,1,1,-1,-1,-1)$ & $(2[-4] 4-1)$ & 11 & \\
\hline$R_{10,4}^{10,3}$ & $\Gamma_{10}^{4}(+-++)$ & $G(1,-1,-1,1,1,1,-1,1,1,-1)$ & $\left(\begin{array}{lllll}-1 & 3 & {[-3]} & 3 & -1\end{array}\right)$ & 11 & \\
\hline$R_{10,4}^{4} !$ & $\Gamma_{10}^{4}(-+--)$ & $G(-1,1,1,-1,-1,-1,1,-1,-1,1)$ & $\left(\begin{array}{lllll}-1 & 3 & {[-3]} & 3 & -1\end{array}\right)$ & 11 & \\
\hline$R_{10,5}^{4}$ & $\Gamma_{10}^{4}(-+++)$ & $G(-1,1,1,1,-1,-1,1,1,-1,1)$ & $\left(\begin{array}{lllll}-1 & 3 & -4 & 3 & {[0]}\end{array}\right)$ & 11 & \\
\hline$R_{10,5}^{10,5} !$ & $\Gamma_{10}^{4}(+---)$ & $G(1,-1,-1,-1,1,1,-1,-1,1,-1)$ & $\left(\left[\begin{array}{lllll}0 & 3 & -4 & 3 & -1\end{array}\right)\right.$ & 11 & \\
\hline$R_{10,6}^{10,5}$ & $\Gamma_{10}^{4}(++--)$ & $G(1,1,-1,-1,1,-1,-1,-1,1,1)$ & 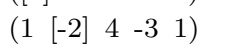 & 11 & \\
\hline$R_{10,6}^{4} !$ & $\Gamma_{10}^{4}(--++)$ & $G(-1,-1,1,1,-1,1,1,1,-1,-1)$ & 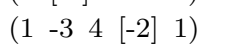 & 11 & \\
\hline$R_{10,7}^{4}$ & $\Gamma_{10}^{4}(+-+-)$ & $G(1,-1,-1,1,1,1,-1,-1,1,-1)$ & $\left(\left[\begin{array}{llll}-1 & 5 & -4 & 1\end{array}\right)\right.$ & 11 & \\
\hline$R_{10,7}^{4} !$ & $\Gamma_{10}^{4}(-+-+)$ & $G(-1,1,1,-1,-1,-1,1,1,-1,1)$ & $(1-4 \quad 5 \quad[-1])$ & 11 & \\
\hline$R_{10,8}^{4}$ & $\Gamma_{10}^{4}(-++-)$ & $G(-1,1,1,1,-1,-1,1,-1,-1,1)$ & $\left(-25\left[\begin{array}{ll}-3 & 1\end{array}\right)\right.$ & 11 & \\
\hline$R_{10,8}^{4}$ ! & $\Gamma_{10}^{4}(+--+)$ & $G(1,-1,-1,-1,1,1,-1,1,1,-1)$ & $(1[-3] 5-2)$ & 11 & \\
\hline
\end{tabular}

The proof of Theorem 4.1 is given in Sects. 5, 6 and 7, where we attempt to find isotopic ribbon 2-knot pairs which are presented by linear Gauss diagrams with up to 4 chords. Actually, Theorem 4.1 does not claim the ribbon 2-knots listed in Tables 2, 3 and 4 are mutually distinct. A pair of ribbon 2-knots with the same Alexander polynomial might be isotopic. In the forthcoming paper [11] we discuss the classification of these ribbon 2-knots. 
Table 3. Cont'd.

\begin{tabular}{|c|c|c|c|c|}
\hline Name & Gauss diagram & Group & $\Delta(t)$ & Det \\
\hline$R_{11,1}^{4}$ & $\Gamma_{11}^{4}(++++)$ & $G(1,1,-1,1,1,1,-1,-1)$ & $\left(\begin{array}{lllll}1 & -2 & {[3]} & -2 & 1\end{array}\right)$ & 9 \\
\hline$R_{11,1}^{4} !$ & $\Gamma_{11}^{4}(----)$ & $G(-1,-1,1,-1,-1,-1,1,1)$ & $\left(\begin{array}{lllll}1 & -2 & {[3]} & -2 & 1\end{array}\right)$ & 9 \\
\hline$R_{11,2}^{4}$ & $\Gamma_{11}^{4}(+++-)$ & $G(1,1,-1,1,1,-1,-1,-1)$ & $([2]-3 \quad 3 r-1)$ & 9 \\
\hline$R_{11,2}^{4} !$ & $\Gamma_{11}^{4}(---+)$ & $G(-1,-1,1,-1,-1,1,1,1)$ & $\left(\begin{array}{llll}-1 & 3 & -3 & {[2]}\end{array}\right)$ & 9 \\
\hline$R_{11,3}^{4}$ & $\begin{array}{l}\Gamma_{11}^{4}(++-+) \\
\Gamma_{11}^{4}(+-+-)\end{array}$ & $G(1,-1,-1,1,1,1,-1,-1)$ & $\left(\begin{array}{llll}-1 & {[4]} & -3 & 1\end{array}\right)$ & 9 \\
\hline$R_{11,3}^{4} !$ & $\begin{array}{l}\Gamma_{11}^{4}(--+-) \\
\Gamma_{11}^{4}(-+-+)\end{array}$ & $G(-1,1,1,-1,-1,-1,1,1)$ & $\left(\begin{array}{llll}1 & -3 & {[4]} & -1\end{array}\right)$ & 9 \\
\hline$R_{11,4}^{4}$ & $\Gamma_{11}^{4}(+-++)$ & $G(-1,1,1,1,-1,1,1,-1)$ & $\left(\begin{array}{lllll}-1 & 2 & -2 & {[3]} & -1\end{array}\right)$ & 9 \\
\hline$R_{11,4}^{4} !$ & $\Gamma_{11}^{4}(-+--)$ & $G(1,-1,-1,-1,1,-1-1,1)$ & $\left(\begin{array}{lllll}-1 & {[3]} & -2 & 2 & -1\end{array}\right)$ & 9 \\
\hline$R_{11,5}^{4}$ & $\Gamma_{11}^{4}(-+++)$ & $G(1,1,-1,-1,1,1,-1,1)$ & $\left(\begin{array}{lll}2 & -4 & {[3]}\end{array}\right)$ & 9 \\
\hline$R_{11,5}^{4}$ ! & $\Gamma_{11}^{4}(+---)$ & $G(-1,-1,1,1,-1,-1,1,-1)$ & $\left(\begin{array}{lll}{[3} & -4 & 2\end{array}\right)$ & 9 \\
\hline$R_{11,6}^{4}$ & $\Gamma_{11}^{4}(++--)$ & $G(1,-1,-1,1,1,-1,-1,-1)$ & $\left(\left[\begin{array}{llll}{[1]} & -2 & 4 & -2\end{array}\right)\right.$ & 9 \\
\hline$R_{11,6}^{4} !$ & $\Gamma_{11}^{4}(--++)$ & $G(-1,1,1,-1,-1,1,1,1)$ & $\left(\begin{array}{llll}-2 & 4 & -2 & {[1]}\end{array}\right)$ & 9 \\
\hline$R_{11,7}^{4}$ & $\Gamma_{11}^{4}(-++-)$ & $G(1,1,-1,-1,1,-1,-1,1)$ & $\left(\begin{array}{llll}-1 & {[4]} & -3 & 1\end{array}\right)$ & 9 \\
\hline$R_{11,7}^{4} !$ & $\Gamma_{11}^{4}(+--+)$ & $G(-1,-1,1,1,-1,1,1,-1)$ & $\left(\begin{array}{llll}1 & -3 & {[4]} & -1\end{array}\right)$ & 9 \\
\hline$R_{12,1}^{4}$ & $\Gamma_{12}^{4}(+++-)$ & $G(1,1,-2,1)$ & $([1])$ & 1 \\
\hline$R_{12,1}^{4}$ ! & $\Gamma_{12}^{4}(---+)$ & $G(-1,-1,2,-1)$ & $([1])$ & 1 \\
\hline$R_{12,2}^{4}$ & $\Gamma_{12}^{4}(-+++)$ & $G(-1,1,2,1)$ & $\left(\begin{array}{lllll}1 & {[-1]} & 0 & 2 & -1\end{array}\right)$ & 1 \\
\hline$R_{12,2}^{4} !$ & $\Gamma_{12}^{4}(+---)$ & $G(1,-1,-2,-1)$ & $\left(\begin{array}{lllll}-1 & 2 & 0 & {[-1]} & 1\end{array}\right)$ & 1 \\
\hline
\end{tabular}

Table 4. Ribbon 2-knots presented by the linear Gauss diagram $\Gamma_{13}^{4}$.

\begin{tabular}{|c|c|c|c|c|}
\hline Name & Gauss diagram & $\Delta(t)$ & Det & \\
\hline$R_{1}^{2} \# R_{1}^{2}$ & $\begin{array}{l}\Gamma_{13}^{4}(++++), \Gamma_{13}^{4}(----) \\
\Gamma_{13}^{4}(++--), \Gamma_{13}^{4}(--++)\end{array}$ & $\left(\begin{array}{lllll}1 & -2 & {[3]} & -2 & 1\end{array}\right)$ & 9 & $\mathrm{a}$ \\
\hline$R_{1}^{2} \# R_{2}^{2}$ & $\begin{array}{l}\Gamma_{13}^{4}(+++-), \Gamma_{13}^{4}(--+-) \\
\Gamma_{13}^{4}(-+--), \Gamma_{13}^{4}(-+++)\end{array}$ & $([2]-3 \quad 3 r-1)$ & 9 & \\
\hline$R_{1}^{2} \# R_{2}^{2 !}$ & $\begin{array}{l}\Gamma_{13}^{4}(++-+), \Gamma_{13}^{4}(---+) \\
\Gamma_{13}^{4}(-+--), \Gamma_{13}^{4}(-+++)\end{array}$ & $\left(\begin{array}{llll}-1 & 3 & -3 & {[2]}\end{array}\right)$ & 9 & \\
\hline$R_{2}^{2} \# R_{2}^{2} !$ & $\Gamma_{13}^{4}(+--+), \Gamma_{13}^{4}(-++-)$ & $(-2[5]-2)$ & 9 & $\mathrm{a}$ \\
\hline$R_{2}^{2} \# R_{2}^{2}$ & $\Gamma_{13}^{4}(+-+-)$ & 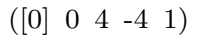 & 9 & \\
\hline$R_{2}^{2} ! \# R_{2}^{2} !$ & $\Gamma_{13}^{4}(-+-+)$ & $\left(\begin{array}{lllll}1 & -4 & 4 & 0 & [0])\end{array}\right)$ & 9 & \\
\hline
\end{tabular}

In the remaining of this section, we explain the calculation of the knot group and Alexander polynomial.

Let $A$ be an oriented virtual arc diagram. Then we can define the group of $A$ in the same way as a virtual knot, which uses a generalization of Wirtinger's algorithm; see [12]. It is also the fundamental group of the complement of the corresponding ribbon 2-knot Tube $(A), \pi_{1}\left(\boldsymbol{R}^{4}-\operatorname{Tube}(A)\right)$ [19, Proposition 5.3]. Furthermore, we can obtain the group presentation of $A$ (and so of Tube $(A)$ ) from a linear Gauss diagram $\gamma$ which presents $A$ as for a virtual knot explained in [2, p. 1049]; cf. [13, 
Sec. 2.3]. If we cut the interval at each arrowhead (forgetting arrowtails), the interval of $\gamma$ is divided into a set of arcs. To each of these arcs there corresponds a generator of the group, which corresponds to a meridian generator of the group of $\operatorname{Tube}(A)$. Each arrow gives rise to a relation. Suppose the sign of an arrow is $p$, its tail lies on an arc labeled $x$, its head is the final point of an arc labeled $y$ and the initial point of an arc labeled $z$. Then we assign to this arrow the relation $z=x^{-p} y x^{p}$, meaning:

$$
x^{-p} y x^{p}= \begin{cases}x^{-1} y x & \text { if } p=+ \\ x y x^{-1} & \text { if } p=-\end{cases}
$$

The resulting group is the group of the virtual arc $A$ and also the group of the corresponding ribbon 2-knot $\operatorname{Tube}(A)$, which we denote by $\pi \gamma$.

Example 4.3. We calculate the group $\pi \Gamma_{6}^{4}(p, q, r, s)$. Cutting the interval at each arrowhead, we obtain 5 arcs. Take generators $x, y, z, u, v$ as shown in Fig. 30. Then we obtain the following relations:

$$
\begin{aligned}
& y=v^{-p} x v^{p}, \\
& z=x^{-q} y x^{q}, \\
& u=x^{-r} z x^{r} \\
& v=z^{-s} u z^{s} .
\end{aligned}
$$

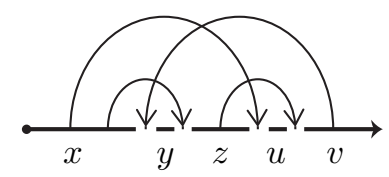

Fig. 30. Generators of the group $\pi \Gamma_{6}^{4}(p, q, r, s)$.

In fact, from the linear Gauss diagram $\Gamma_{6}^{4}(+--+)$ we obtain a virtual arc diagram as shown in Fig. 31, from which we obtain the above group presentation by Wirtinger's algorithm.

Substitute (4.4) and (4.6) into (4.5) and (4.7), respectively. Then we obtain

$$
\begin{aligned}
& z=x^{-q} v^{-p} x v^{p} x^{q}, \\
& v=z^{-s} x^{-r} z x^{r} z^{s} .
\end{aligned}
$$

Substitute (4.8) into (4.9). Then we obtain

$$
\begin{aligned}
v & =x^{-q} v^{-p} x^{-s} v^{p} x^{q} x^{-r} x^{-q} v^{-p} x v^{p} x^{q} x^{r} x^{-q} v^{-p} x^{s} v^{p} x^{q} \\
& =\left(x^{-q} v^{-p} x^{-s} v^{p} x^{-r} v^{-p}\right) x\left(v^{p} x^{r} v^{-p} x^{s} v^{p} x^{q}\right) .
\end{aligned}
$$

So, the group $\pi \Gamma_{6}^{4}(p, q, r, s)$ is presented by $G(-q,-p,-s, p,-r,-p)$. 


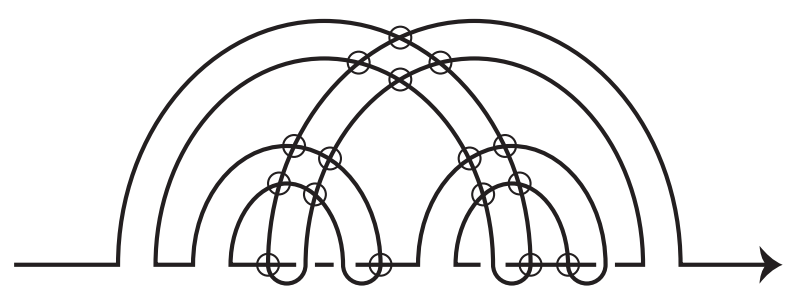

Fig. 31. Virtual arc diagram with Gauss diagram $\Gamma_{6}^{4}(+--+)$

Alternatively, substitute (4.9) into (4.4). Then we obtain

$$
y=z^{-s} x^{-r} z^{-p} x^{r} z^{s} x z^{-s} x^{-r} z^{p} x^{r} z^{s}
$$

Then substitute (4.11) into (4.5). Then we obtain

$$
z=\left(x^{-q} z^{-s} x^{-r} z^{-p} x^{r} z^{s}\right) x\left(z^{-s} x^{-r} z^{p} x^{r} z^{s} x^{q}\right) .
$$

So, the group $\pi \Gamma_{6}^{4}(p, q, r, s)$ is also presented by $G(-q,-s,-r,-p, r, s)$. In Table 3 we list the former presentation. In particular, the group $\pi \Gamma_{6}^{4}(+--+)$ has two presentations, $G(1,-1,-1,1,1,-1)$ and $G(1,-1,1,-1,-1,1)$. It is not trivial that they present the same group.

Example 4.4. We calculate the group $\pi \Gamma_{11}^{4}(p, q, r, s)$. Take generators $x, y, z, u$, $v$ as shown in Fig. 32. Then we obtain the following relations:

$$
\begin{aligned}
& y=z^{-p} x z^{p}, \\
& z=v^{-q} y v^{q}, \\
& u=y^{-r} z y^{r}, \\
& v=x^{-s} u x^{s} .
\end{aligned}
$$

Removing $x, y$ and $u$, we obtain:

$$
v=\left(z^{p} v^{q} z^{-s} v^{-q} z^{-p}\right)\left(v^{q} z^{-r} v^{-q} z v^{q} z^{r} v^{-q}\right)\left(z^{p} v^{q} z^{s} v^{-q} z^{-p}\right) .
$$

So, the group $\pi \Gamma_{11}^{4}(p, q, r, s)$ is presented by $G(p, q,-s,-q,-p, q,-r,-q)$, which is isomorphic to $G(q, r,-q, p, q, s,-q,-p)$. In Table 3 we list this presentation.

Alternatively, removing $y, u$ and $v$, we obtain:

$$
z=\left(x^{-s} z^{-p} x^{-r} z^{-q} x^{r} z^{p} x^{s} z^{-p}\right) x\left(z^{p} x^{-s} z^{-p} x^{-r} z^{q} x^{r} z^{q} x^{s}\right) .
$$

So, the group $\pi \Gamma_{11}^{4}(p, q, r, s)$ is also presented by $G(-s,-p,-r,-q, r, p, s,-p)$.

From the group presentation Eq. (4.1) we obtain the Alexander polynomial as follows:

$$
\begin{array}{r}
1-t^{a_{1}}+t^{a_{1}+b_{1}}-t^{a_{1}+b_{1}+a_{2}}+t^{a_{1}+b_{1}+a_{2}+b_{2}}-\cdots \\
-t^{a_{1}+b_{1}+\cdots+b_{n-1}+a_{n}}+t^{a_{1}+b_{1}+\cdots+b_{n-1}+a_{n}+b_{n}}
\end{array}
$$




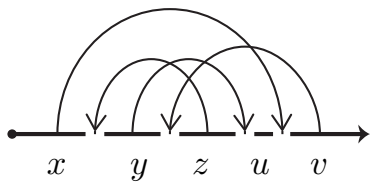

Fig. 32. Generators $x, y, z, u, v$ of the group $\pi \Gamma_{11}^{4}(p, q, r, s)$.

see [15]. If $\Delta(t)$ is the normalized Alexander polynomial of a ribbon 2-knot, then the normalized Alexander polynomial of its mirror image is given by $\Delta\left(t^{-1}\right)$, and thus their determinants coincide. Further, if $\Delta(t) \neq \Delta\left(t^{-1}\right)$, then it is not amphicheiral.

Remark 4.5. We can define a sequence of invariants for a ribbon 2-knot by $\alpha_{n}(K)=(l / n !) d^{n} / d t^{n} \Delta_{K}(1)$, which are finite type invariants [3, Theorem2.2]; see also $[4,10]$.

\section{Ribbon 2-Knots Presented by Linear Gauss Diagrams with up to 3 Chords}

In this section, we prove Theorem 4.1 for a linear Gauss diagram with up to 3 chords.

\subsection{Linear Gauss diagrams with up to 2 chords}

A ribbon 2-knot presented by a linear Gauss diagram with one chord is trivial by the move $A_{I}$. In order to enumerate ribbon 2-knots presented by linear Gauss diagrams with 2 chords, by Lemma 3.6 and the move $\mathrm{E}_{\mathrm{II}}$ we have only to consider the diagram $\Gamma_{1}^{2}$ as shown in Fig. 29. Then since $\Gamma_{1}^{2}(++) \stackrel{\mathrm{r}}{\sim} \Gamma_{1}^{2}(--)$ by the reversing move, we obtain the ribbon 2 -knots $R_{1}^{2}, R_{2}^{2}, R_{2}^{2}$ ! as listed in Table 2 . The group is presented as follows:

$$
\pi \Gamma_{1}^{2}(p, q)=G(p, q)
$$

\subsection{Linear Gauss diagrams with 3 chords}

We enumerate ribbon 2-knots presented by linear Gauss diagrams with 3 chords.

Lemma 5.1. Any linear Gauss diagram with 3 chords is r-equivalent to either the trivial diagram or the diagrams $\Gamma_{1}^{2}, \Gamma_{1}^{3}$, or $\Gamma_{2}^{3}$ given in Fig. 29 with some signs.

Proof. By Lemma 3.6 we have only to consider three linear Gauss diagrams $\beta_{i}$, $i=1,2,3$, as shown in Fig. 33, where we ignore the orientation of the interval, and the orientations and signs of the chords.

Then, by the move $\mathrm{E}_{\mathrm{II}}$ and reversing move we have only to consider the linear Gauss diagrams $\Gamma_{1}^{3}, \Gamma_{2}^{3}, \beta_{21}, \beta_{31}$ as shown in Fig. 34. By the move D we have 


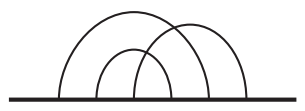

$\beta_{1}$

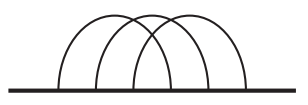

$\beta_{2}$

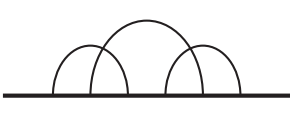

$\beta_{3}$

Fig. 33. Unoriented linear Gauss diagrams with 3 chords.

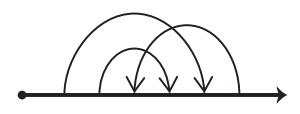

$\Gamma_{1}^{3}$

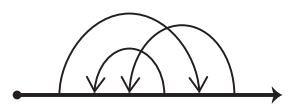

$\Gamma_{2}^{3}$

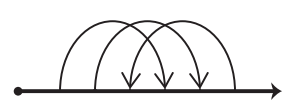

$\beta_{21}$

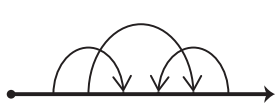

$\beta_{31}$

Fig. 34. Oriented linear Gauss diagrams with 3 chords.

$\beta_{21}(p, q, r) \stackrel{\mathrm{r}}{\sim} \Gamma_{1}^{3}(p, q, r)$, and by the moves $\mathrm{D}$ and $\mathrm{A}_{\mathrm{I}}$ we have $\beta_{31}(p, q, r) \stackrel{\mathrm{r}}{\sim} \Gamma_{1}^{2}(q, r)$. This completes the proof.

Now, we consider the diagrams $\Gamma_{1}^{3}(p, q, r)$ and $\Gamma_{2}^{3}(p, q, r)$. By the moves $\mathrm{A}_{\mathrm{II}}$ and $\mathrm{A}_{\mathrm{I}}$ the diagram $\Gamma_{1}^{3}(p, q,-q)$ is deformed into the trivial diagram. By the move $\Omega_{5}$ and reversing move the diagram $\Gamma_{2}^{3}(p, q, r)$ is deformed into $\Gamma_{2}^{3}(p,-r,-q)$; see Fig. 35(a). This implies $\Gamma_{2}^{3}(+++) \stackrel{\mathrm{r}}{\sim} \Gamma_{2}^{3}(+--)$. Furthermore, by the reversing move and the move $\Omega_{2}$ the diagram $\Gamma_{2}^{3}(p,-p, q)$ is deformed into $\Gamma_{2}^{3}(-q, q,-p)$; see Fig. 35(b). This implies $\Gamma_{2}^{3}(+-+) \stackrel{\mathrm{r}}{\sim} \Gamma_{2}^{3}(-+-)$. Then we obtain the ribbon 2 -knots $R_{i}^{3}, R_{i}^{3}$ ! $(i=1,2,3,4)$ and $R_{5}^{3}$ as listed in Table 2 . The groups are presented as follows:

$$
\begin{aligned}
& \pi \Gamma_{1}^{3}(p, q, r)=G(p, q+r) ; \\
& \pi \Gamma_{2}^{3}(p, q, r)=G(p,-r, q, r) .
\end{aligned}
$$

(a)
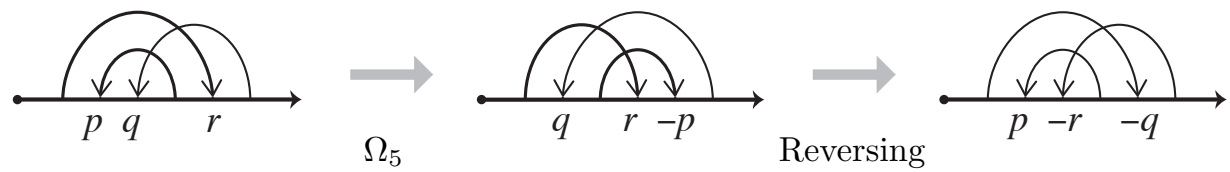

(b)
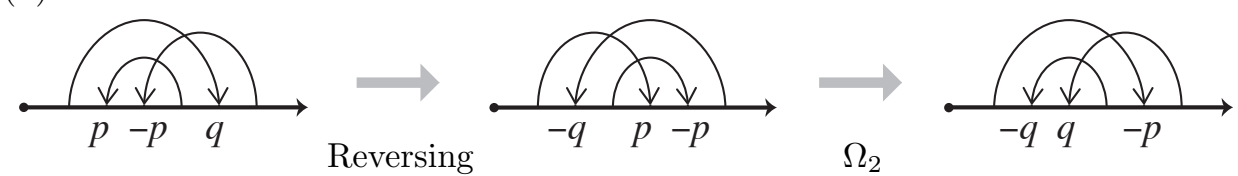

Fig. 35. r-Equivalent linear Gauss diagrams with 3 chords. 


\section{Linear Gauss diagrams with 4 chords}

In this section, we prove the following lemma.

Lemma 6.1. Any linear Gauss diagram with 4 chords is r-equivalent to either a linear Gauss diagram with up to 3 chords or $\Gamma_{l}^{4}, 1 \leq l \leq 13$, given in Fig. 29 with some signs.

Proof. First we note there are seven types of circular Gauss diagrams with 4 chords that has no separated single chord as shown in Fig. 36.

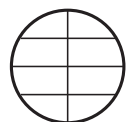

$\gamma_{1}$

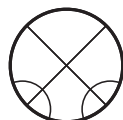

$\gamma_{2}$

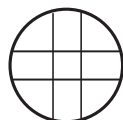

$\gamma_{3}$

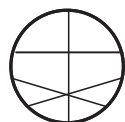

$\gamma_{4}$

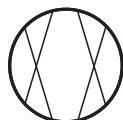

$\gamma_{5}$

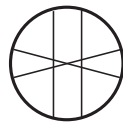

$\gamma_{6}$

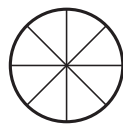

$\gamma_{7}$

Fig. 36. Circular Gauss diagrams with 4 chords.

From the circular Gauss diagram $\gamma_{i}, 1 \leq i \leq 7$, we obtain linear Gauss diagrams $\gamma_{i j}$ ignoring the orientation of the interval, and the orientations and signs of the chords as shown in Fig. 37.

From $\gamma_{i j}$ we obtain oriented Gauss diagrams $\gamma_{i j k}$ as shown in Fig. 38 up to r-equivalence, where:

- $\gamma_{i j k}=\Gamma_{l}^{4}$ means that the diagram $\gamma_{i j k}$ is the same as $\Gamma_{l}^{4}$ given in Fig. 29.

- $\gamma_{i j k} \rightarrow \Gamma^{3}$ means that by the move D the diagram $\gamma_{i j k}(p, q, r, s)$ with any signs $(p, q, r, s= \pm)$ is deformed into a linear Gauss diagram having a single separated chord, which is r-equivalent to a Gauss diagram with up to 3 chords by Lemma 3.6.

- $\gamma_{i j k} \rightarrow \pm \Gamma_{l}^{4}$ means that by the move $\mathrm{D}$ the diagram $\gamma_{i j k}(p, q, r, s)$ is deformed into the Gauss diagram $\pm \Gamma_{l}^{4}\left(p^{\prime}, q^{\prime}, r^{\prime}, s^{\prime}\right)$ for some $l$ and some signs $p^{\prime}, q^{\prime}, r^{\prime}$, $s^{\prime}= \pm$, where $\Gamma_{l}^{4}$ is given in Fig. 29.

- $\gamma_{i j k} \rightarrow \pm \gamma_{i^{\prime} j^{\prime} k^{\prime}}$ means that by the move $\mathrm{D}$ the diagram $\gamma_{i j k}(p, q, r, s)$ is deformed into the Gauss diagram $\pm \gamma_{i^{\prime} j^{\prime} k^{\prime}}\left(p^{\prime}, q^{\prime}, r^{\prime}, s^{\prime}\right)$.

The following are proved by the move $\Omega_{i}, i=5,6,7,8$ :

$$
\begin{array}{ll}
\gamma_{112}(p, q, r, s) \stackrel{\mathrm{r}}{\sim} \Gamma_{7}^{4}(q, r, s,-p) & \text { (by the move } \left.\Omega_{6}\right), \\
\gamma_{113}(p, q, r, s) \stackrel{\mathrm{r}}{\sim} \Gamma_{6}^{4}(q, r, s,-p) & \text { (by the move } \left.\Omega_{5}\right), \\
\gamma_{114}(p, q, r, s) \stackrel{\mathrm{r}}{\sim} \Gamma_{5}^{4}(q, r, s,-p) & \text { (by the move } \left.\Omega_{5}\right), \\
\gamma_{441}(p, q, r, s) \stackrel{\mathrm{r}}{\sim} \Gamma_{6}^{4}(p, s, q, r) & \text { (by the move } \left.\Omega_{8}\right), \\
\gamma_{442}(p, q, r, s) \stackrel{\mathrm{r}}{\sim} \Gamma_{2}^{4}(p, s, q, r) & \text { (by the move } \left.\Omega_{8}\right),
\end{array}
$$




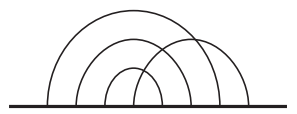

$\gamma_{11}$

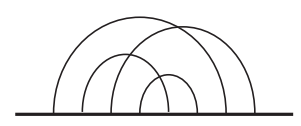

$\gamma_{23}$

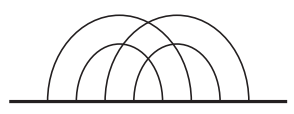

$\gamma_{32}$

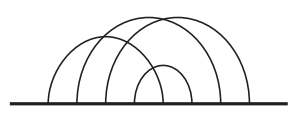

$\gamma_{44}$

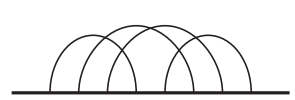

$\gamma_{61}$

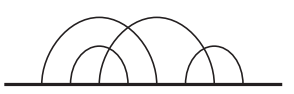

$\gamma_{12}$

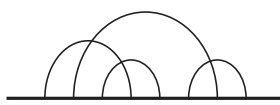

$\gamma_{24}$

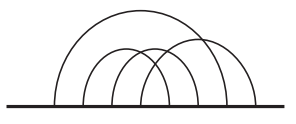

$\gamma_{41}$

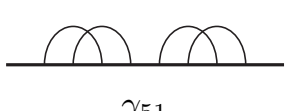

$\gamma_{51}$

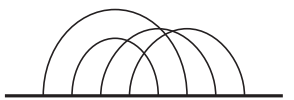

$\gamma_{62}$

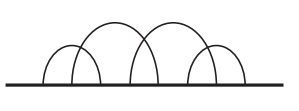

$\gamma_{21}$

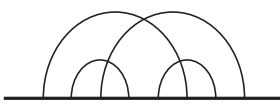

$\gamma_{25}$

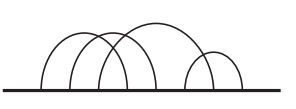

$\gamma_{42}$

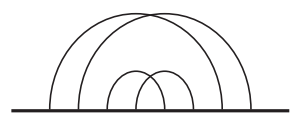

$\gamma_{52}$

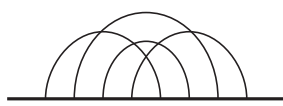

$\gamma_{63}$

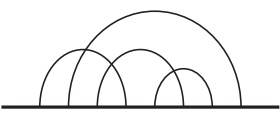

$\gamma_{22}$

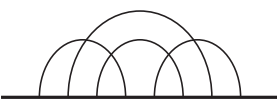

$\gamma_{31}$

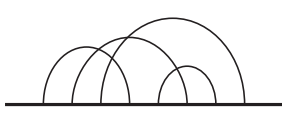

$\gamma_{43}$

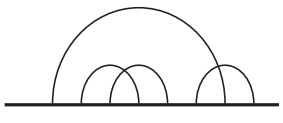

$\gamma_{53}$

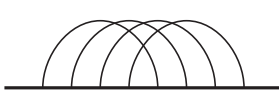

$\gamma_{71}$

Fig. 37. Linear Gauss diagrams $\gamma_{i j}$ obtained from $\gamma_{i}, 1 \leq i \leq 7$.

$$
\begin{array}{ll}
\gamma_{531}(p, q, r, s) \stackrel{\mathrm{r}}{\sim} \Gamma_{8}^{4}(r, p, q, s) & \text { (by the move } \left.\Omega_{7}\right), \\
\gamma_{423}(p, q, r, s) \stackrel{\mathrm{r}}{\sim} \gamma_{243}(p, q, r, s) & \text { (by the move } \left.\Omega_{7}\right), \\
\gamma_{622}(p, q, r, s) \stackrel{\mathrm{r}}{\sim} \gamma_{113}(p, q, s, r) & \text { (by the move } \left.\Omega_{8}\right), \\
\gamma_{713}(p, q, r, s) \stackrel{\mathrm{r}}{\sim} \gamma_{442}(q, p, r, s) & \text { (by the move } \left.\Omega_{7}\right) .
\end{array}
$$

Thus, each linear Gauss diagram $\gamma_{i j k}(p, q, r, s)$ is r-equivalent to either a diagram with up to 3 chords or $\Gamma_{l}^{4}\left(p^{\prime}, q^{\prime}, r^{\prime}, s^{\prime}\right)$ for some $l$ and some signs $p^{\prime}, q^{\prime}, r^{\prime}, s^{\prime}$. This completes the proof.

\section{Ribbon 2-Knots Obtained from the Linear Gauss Diagrams $\Gamma_{l}^{4}$}

In this section, we prove Theorem 4.1 for a linear Gauss diagram with 4 chords using Lemma 6.1; we examine the associated ribbon 2 -knot for each $\Gamma_{l}^{4}(p, q, r, s)$, $1 \leq l \leq 13$.

\subsection{Ribbon 2-knots obtained from $\Gamma_{1}^{4}$}

By the move $\mathrm{A}_{\mathrm{II}}$ if either $q=-r$ or $r=-s$, then $\Gamma_{1}^{4}(p, q, r, s)$ is r-equivalent to a diagram with 2 chords. So, we consider the case $q=r=s$. Then we obtain the 


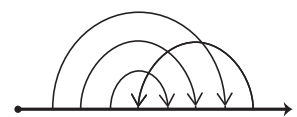

$\gamma_{111}=\Gamma_{1}^{4}$

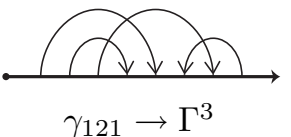

$\gamma_{121} \rightarrow \Gamma^{3}$

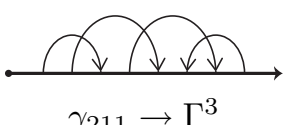

$\gamma_{211} \rightarrow \Gamma^{3}$

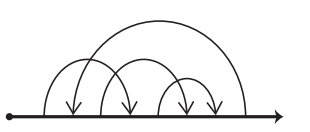

$\gamma_{221}=\Gamma_{10}^{4}$

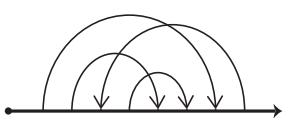

$\gamma_{231}$
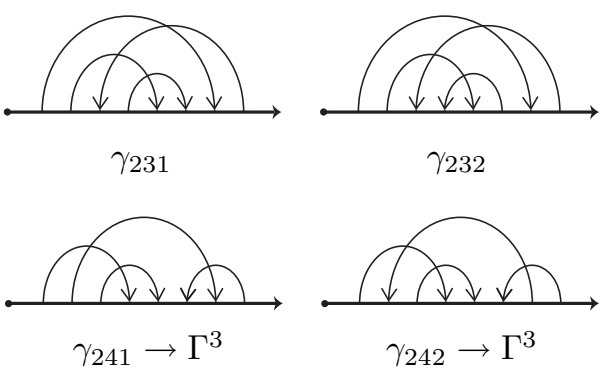

$\gamma_{232}$
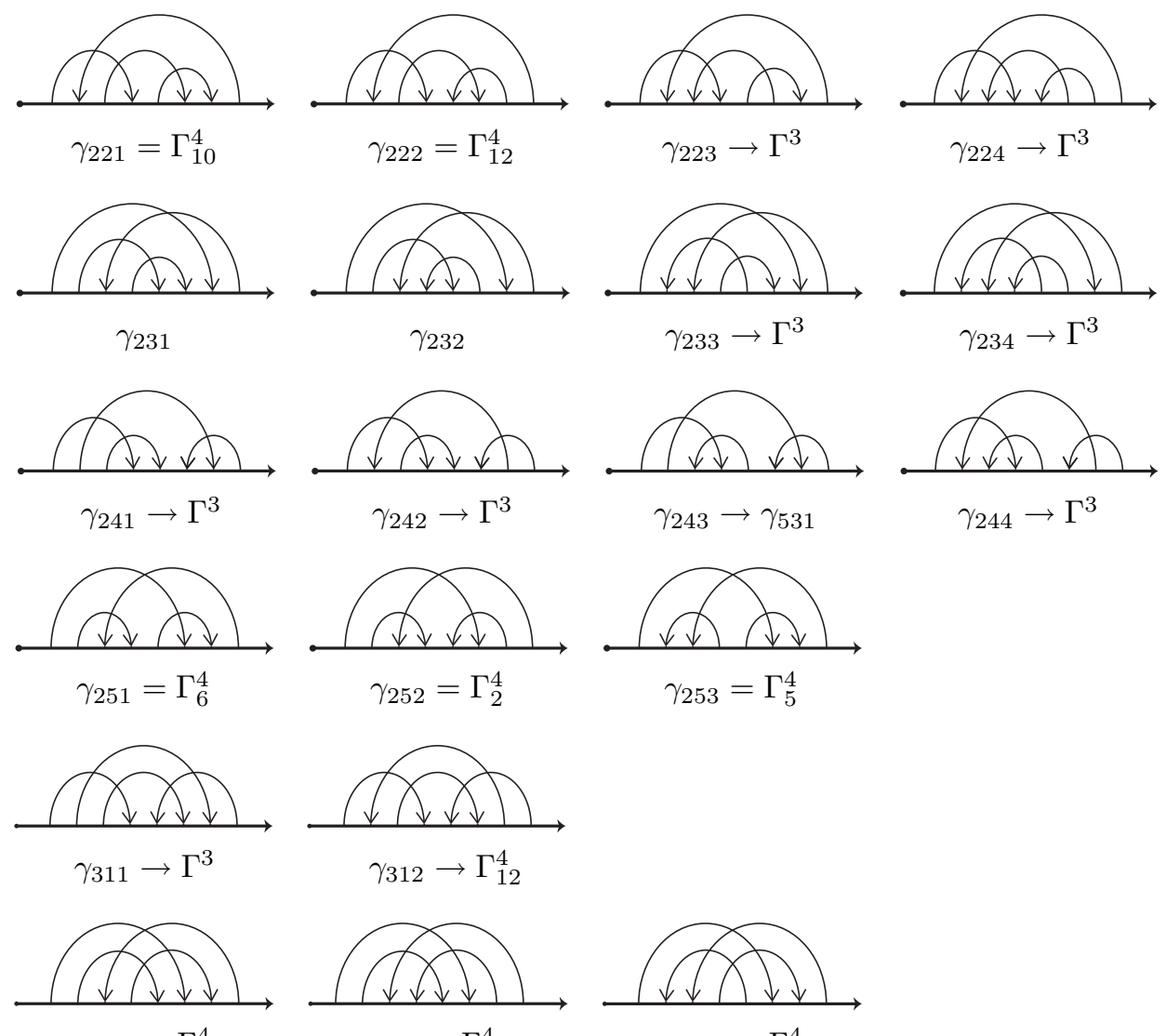

$\gamma_{321}=\Gamma_{7}^{4}$
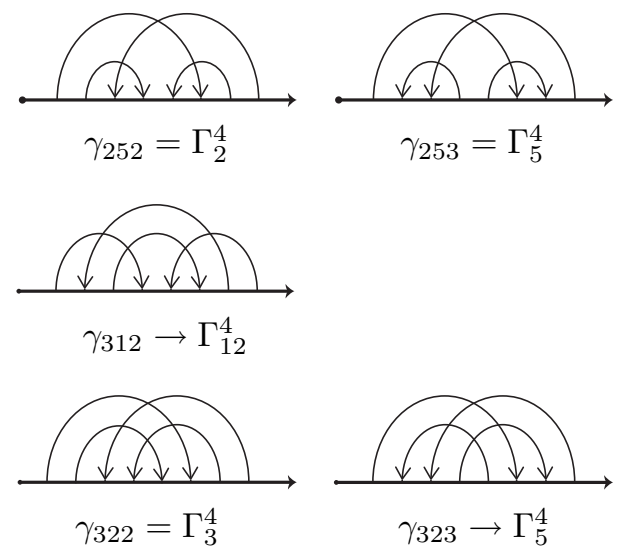

Fig. 38. Oriented linear Gauss diagrams $\gamma_{i j k}$ obtained from $\gamma_{i j}$ 


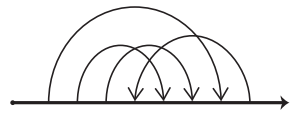

$\gamma_{411} \rightarrow \Gamma_{1}^{4}$

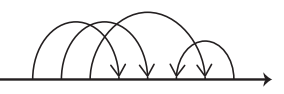

$\gamma_{421} \rightarrow \Gamma^{3}$

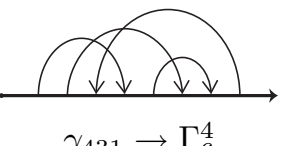

$\gamma_{431} \rightarrow \Gamma_{6}^{4}$

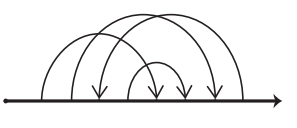

$\gamma_{441} \rightarrow \gamma_{231}$
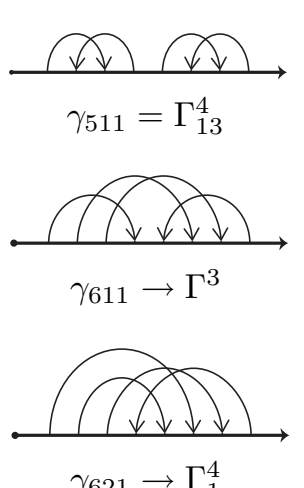

$\gamma_{621} \rightarrow \Gamma_{1}^{4}$

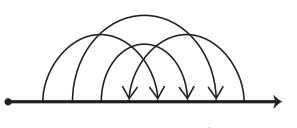

$\gamma_{631} \rightarrow \Gamma_{1}^{4}$

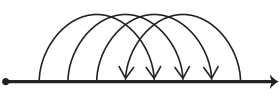

$\gamma_{711} \rightarrow \Gamma_{1}^{4}$
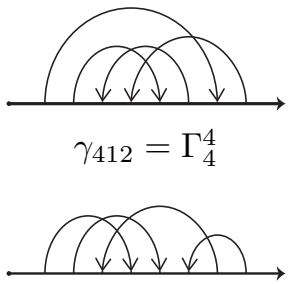

$\gamma_{422} \rightarrow \Gamma^{3}$
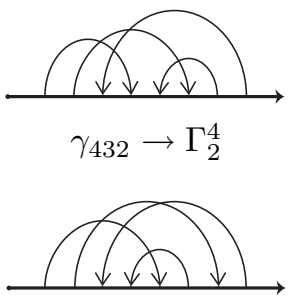

$\gamma_{442}$
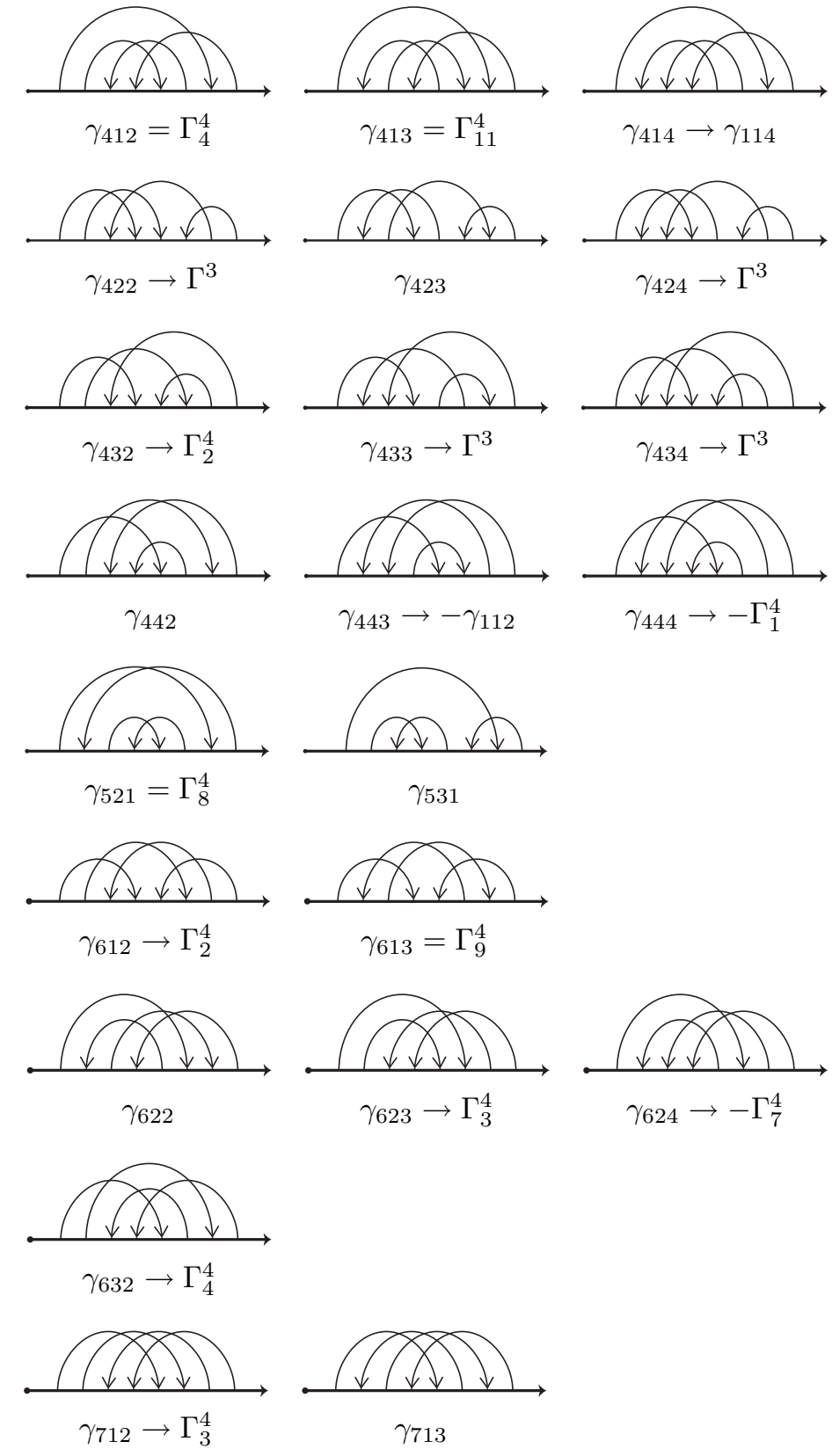

Fig. 38. Cont'd.

ribbon 2 -knots $R_{1, j}^{4}, R_{1, j}^{4} !, j=1,2$, as listed in Table 3 . The group is presented as 
follows:

$$
\pi \Gamma_{1}^{4}(p, q, r, s)=G(p, q+r+s)
$$

\subsection{Ribbon 2-knots obtained from $\Gamma_{2}^{4}$}

By the move $\mathrm{D}$ the diagram $\Gamma_{2}^{4}(p, q, r, s)$ is deformed into $\gamma_{612}(p, q, r, s)$ given in Fig. 38. If either $s=-q$ or $r=-p$, then by the move $\mathrm{A}_{\text {III }}$ the diagram $\gamma_{612}(p, q, r, s)$ is deformed into a diagram with a single separated chord; see Fig. 39. So, we consider the case $s=q$ and $r=p$. Note that $\Gamma_{2}^{4}(++++) \stackrel{\mathrm{r}}{\sim} \Gamma_{2}^{4}(----)$ by the reversing move. Then we obtain the ribbon 2 -knots $R_{2,1}^{4}, R_{2,2}^{4}, R_{2,2}^{4}$ ! as listed in Table 3 . The group is presented as follows:

$$
\pi \Gamma_{2}^{4}(p, q, r, s)=G(p, q, r, s) .
$$
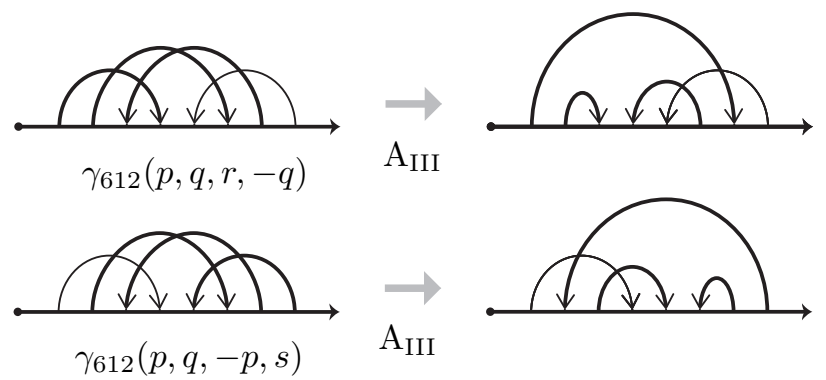

Fig. 39. The diagrams $\gamma_{612}(p, q, r,-q)$ and $\gamma_{612}(p, q,-p, s)$ are deformed into diagrams with a single separated chord.

\subsection{Ribbon 2-knots obtained from $\Gamma_{3}^{4}$}

If either $p=-q$ or $q=-r$, then by the move $\mathrm{A}_{\mathrm{II}}$ the diagram $\Gamma_{3}^{4}(p, q, r, s)$ is r-equivalent to a diagram with 2 chords. So, we consider the case $p=q$ and $r=s$. Note that $\Gamma_{3}^{4}(++++) \stackrel{\mathrm{r}}{\sim} \Gamma_{3}^{4}(----)$ by the reversing move. Then we obtain the ribbon 2-knots $R_{3,1}^{4}, R_{3,2}^{4}, R_{3,2}^{4}$ ! as listed in Table 3 . The group is presented as follows:

$$
\pi \Gamma_{3}^{4}(p, q, r, s)=G(p+q, r+s)
$$

\subsection{Ribbon 2-knots obtained from $\Gamma_{4}^{4}$}

By the move $\mathrm{D}$ the diagram $\Gamma_{4}^{4}(p, q, r, s)$ is deformed into $\gamma_{632}(p, q, r, s)$ given in Fig. 38. Then if $s=-r$, by the move $\mathrm{A}_{\mathrm{III}}$ the diagram $\gamma_{632}(p, q, r, s)$ is deformed into a Gauss diagram with a single separated chord; see Fig. 40. So, we consider 
the cases $r=s$. Then we obtain the ribbon 2 -knots $R_{4, j}^{4}, R_{4, j}^{4} !, j=1,2,3,4$, as listed in Table 3 . The group is presented as follows:

$$
\pi \Gamma_{4}^{4}(p, q, r, s)=G(p,-s, q, r+s) .
$$
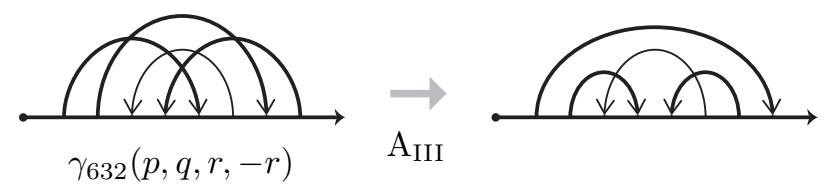

$\mathrm{A}_{\text {III }}$

Fig. 40. $\gamma_{632}(p, q, r,-r)$ is r-equivalent to a diagram with a single separated chord.

\subsection{Ribbon 2-knots obtained from $\Gamma_{5}^{4}$}

By the move $\Omega_{5}$ the diagram $\Gamma_{5}^{4}(p, q, r, s)$ is r-equivalent to $\gamma_{114}(-s, p, q, r)$ given in Fig. 38; see Fig. 41. If $p=s$, then by the move $\mathrm{A}_{\mathrm{II}}$ the diagram $\gamma_{114}(-s, p, q, r)$ is r-equivalent to a diagram with 2 chords. So, we consider the case $p=-s$. Note that by the reversing move we have $\Gamma_{5}^{4}(+---) \stackrel{\mathrm{r}}{\sim} \Gamma_{5}^{4}(+++-)$ and $\Gamma_{5}^{4}(-+++) \stackrel{\mathrm{r}}{\sim} \Gamma_{5}^{4}(--$ $-+)$.

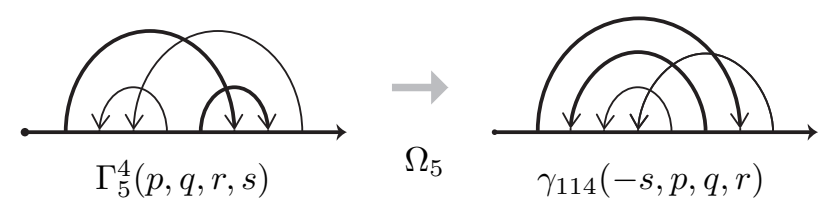

Fig. 41. The diagram $\Gamma_{6}^{4}(p, q, r, s)$ is deformed into $\gamma_{114}(-s, p, q, r)$.

Furthermore, we have the following r-equivalent pairs.

\section{Lemma 7.1.}

$$
\begin{aligned}
& \Gamma_{4}^{4}(+-++) \stackrel{\mathrm{r}}{\sim} \Gamma_{5}^{4}(-+-+) \\
& \Gamma_{4}^{4}(-+--) \stackrel{\mathrm{r}}{\sim} \Gamma_{5}^{4}(+-+-) \\
& \Gamma_{4}^{4}(-+++) \stackrel{\mathrm{r}}{\sim} \Gamma_{5}^{4}(---+) \\
& \Gamma_{4}^{4}(+---) \stackrel{\mathrm{r}}{\sim} \Gamma_{5}^{4}(+++-)
\end{aligned}
$$

Proof. Figure 42 shows the diagrams $\Gamma_{4}^{4}(p,-p,+,+)$ and $\Gamma_{5}^{4}(-,-, p,+)$ are $\mathrm{r}-$ equivalent to the same diagram, proving Eqs. (7.5) and (7.7); Eqs. (7.6) and (7.8) are their mirror images. 
Then the remaining diagrams are $\Gamma_{5}^{4}(--++)$ and $\Gamma_{5}^{4}(++--)$, which present the ribbon 2-knots $R_{5,1}^{4}$ and $R_{5,1}^{4}$ !, respectively, as listed in Table 3. The group is presented as follows:

$$
\pi \Gamma_{5}^{4}(p, q, r, s)=G(-r,-q, r, s-p) .
$$

\subsection{Ribbon 2-knots obtained from $\Gamma_{6}^{4}$}

First we show the following lemma.

Lemma 7.2.

$$
\Gamma_{6}^{4}(p, q, r,-q) \stackrel{\mathrm{r}}{\sim} \Gamma_{9}^{4}(p, r, p, q)
$$

Proof. See Fig. 43.

Now we show the following r-equivalence.

\section{Lemma 7.3.}

$$
\begin{aligned}
& \Gamma_{6}^{4}(+++-) \stackrel{\mathrm{r}}{\sim} \Gamma_{6}^{4}(---+) ; \\
& \Gamma_{6}^{4}(--+-) \stackrel{\mathrm{r}}{\sim} \Gamma_{6}^{4}(++--) \stackrel{\mathrm{r}}{\sim} \Gamma_{6}^{4}(+-++) ; \\
& \Gamma_{6}^{4}(++-+) \stackrel{\mathrm{r}}{\sim} \Gamma_{6}^{4}(--++) \stackrel{\mathrm{r}}{\sim} \Gamma_{6}^{4}(-+--) .
\end{aligned}
$$

Proof. First, we show Eq. (7.11). By Lemma 7.2 we have $\Gamma_{6}^{4}(+++-) \stackrel{\text { r }}{\sim} \Gamma_{9}^{4}(++$ $++)$ and $\Gamma_{6}^{4}(---+) \stackrel{\mathrm{r}}{\sim} \Gamma_{9}^{4}(----)$. Since $\Gamma_{9}^{4}(++++) \stackrel{\mathrm{r}}{\sim} \Gamma_{9}^{4}(----)$ by the reversing move, we obtain Eq. (7.11).

Next, we show Eq. (7.13); Eq. (7.12) is its mirror image. We start with the ribbon handlebody $H_{0}$ as shown in Fig. 44, which is consisting of four 0-handles and three 1-handles. We deform this into three ribbon handlebodies $H_{1}, H_{2}, H_{3}$ as shown in Fig. 44 by the stably equivalent move introduced by Marumoto [16, Fig. 22], and so these four ribbon handlebodies represent the same ribbon 2-knot. Then $H_{1}, H_{2}, H_{3}$ are represented by the virtual arcs $A_{1}, A_{2}, A_{3}$, and their diagrams are $\delta_{1}, \delta_{2}, \delta_{3}$, respectively, as shown in Fig. 45. Note that $\delta_{1}=\gamma_{441}(+-++)$, $\delta_{2}=-\gamma_{441}(+--+), \delta_{3}=\gamma_{441}(---+)$. Then $\delta_{1}$ is r-equivalent to $\Gamma_{6}^{4}(++-+)$ by the move $\Omega_{8} ; \delta_{2}$ is r-equivalent to $\gamma_{441}(-++-)$ by the reversing move, which is r-equivalent to $\Gamma_{6}^{4}(--++)$ by the move $\Omega_{8} ; \delta_{3}$ is r-equivalent to $\Gamma_{6}^{4}(-+--)$ by the move $\Omega_{8}$; see Eq. (6.4). Thus we obtain Eq. (7.13). This completes the proof. $\square$

Therefore, we obtain the ribbon 2-knots $R_{6, j}^{4} R_{6, j}^{4} !, j=1,3,4,5,6$, and $R_{6,2}^{4}$ as listed in Table 3. For the group $\pi \Gamma_{6}^{4}(p, q, r, s)$, see Example 4.3.

Remark 7.4. In [24, Example 4.1] Yasuda has shown the ribbon 2-knot represented by $H_{0}$ has three different ribbon handlebodies consisting of two 0-handles and one 1-handle. The ribbon handlebodies $H_{0}$ and $H_{1}$ in Fig. 44 are given in [24, Fig. 7]. 


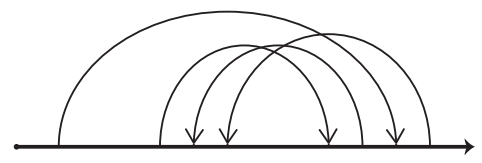

$\Gamma_{4}^{4}(p, q,+,+)$
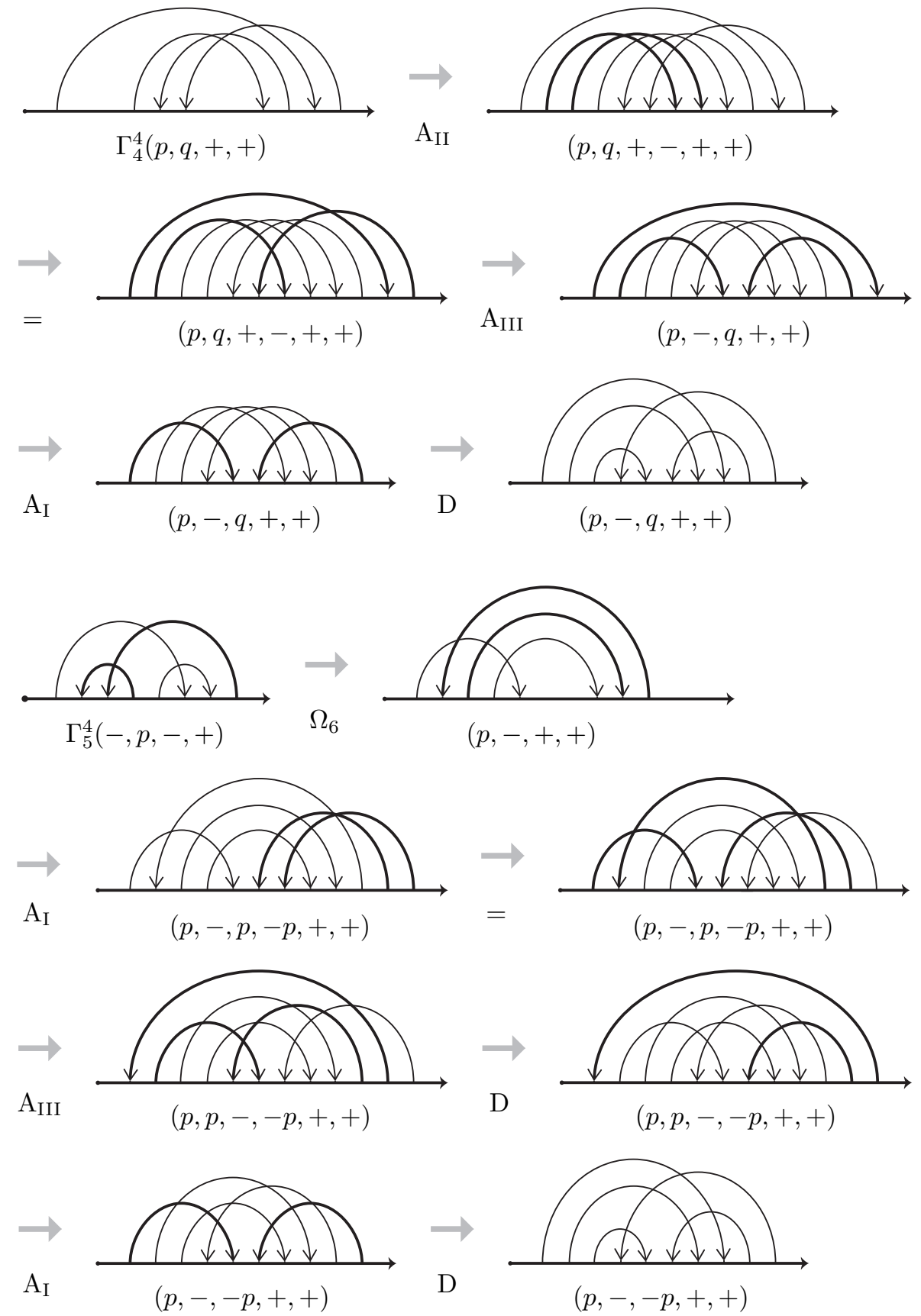

Fig. 42. Proof of Lemma 7.1. 


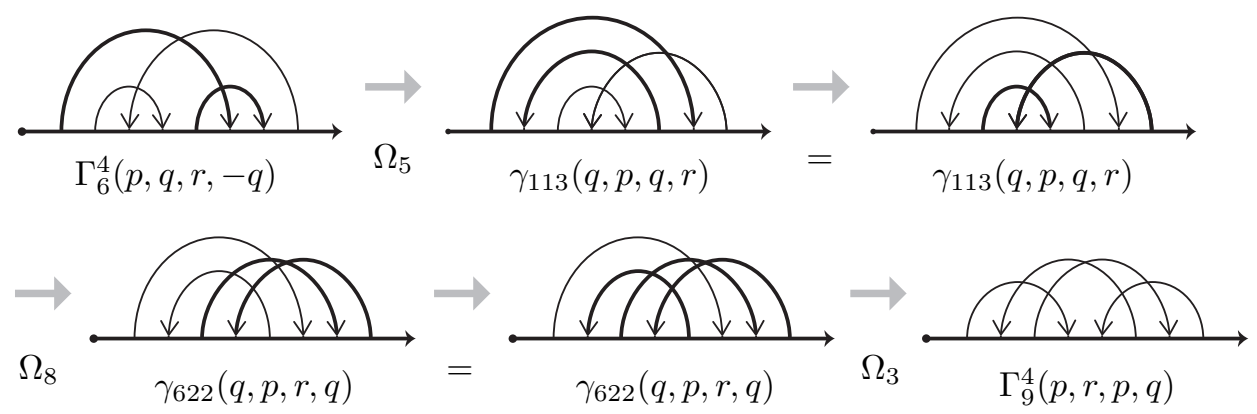

Fig. 43. Proof of Lemma 7.2.

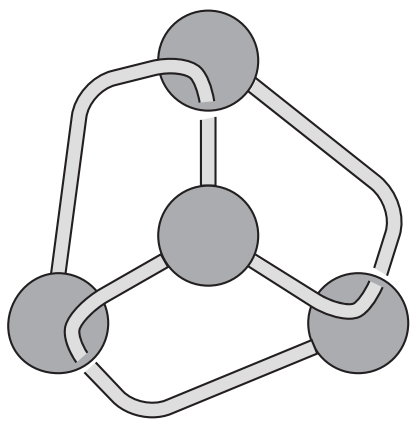

$H_{0}$

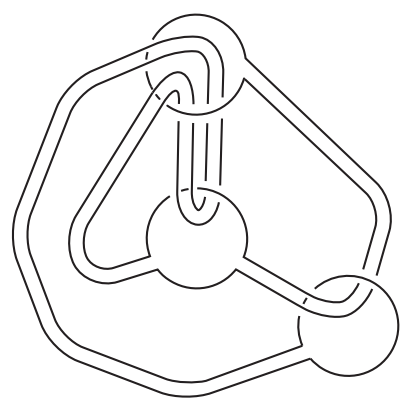

$H_{2}$

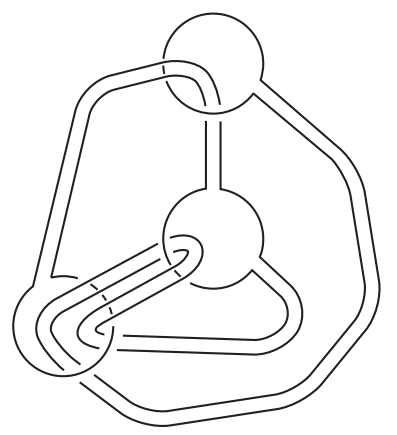

$H_{1}$

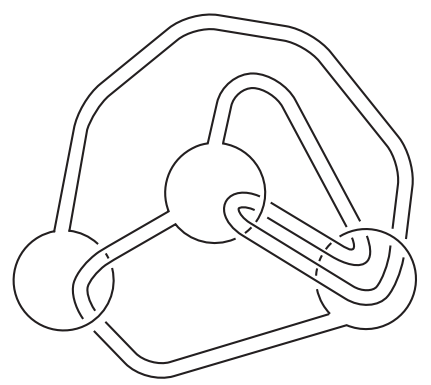

$H_{3}$

Fig. 44. Ribbon handlebodies.

\subsection{Ribbon 2-knots obtained from $\Gamma_{7}^{4}$}

If $q=-r$, then the diagram $\Gamma_{7}^{4}(p, q, r, s)$ is r-equivalent to a diagram with 2 chords by the move $\mathrm{A}_{\mathrm{II}}$. So, we consider the case $q=r$. Then we obtain the ribbon 2-knots 


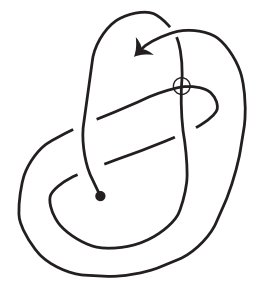

$A_{1}$

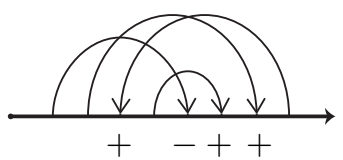

$\delta_{1}$

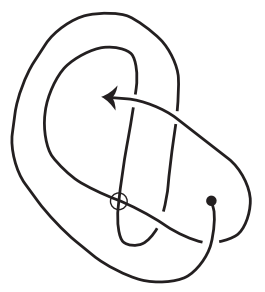

$A_{2}$

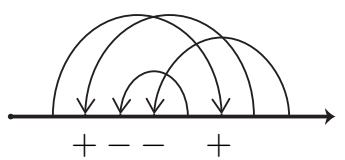

$\delta_{2}$

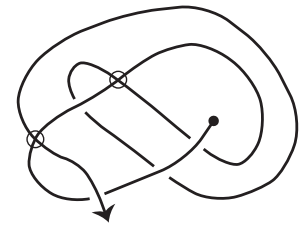

$A_{3}$

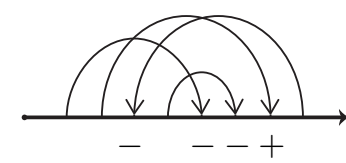

$\delta_{3}$

Fig. 45. Virtual arcs $A_{1}, A_{2}, A_{3}$ representing ribbon handlebodies $H_{1}, H_{2}, H_{3}$, and their diagrams $\delta_{1}, \delta_{2}, \delta_{3}$.

$R_{7, j}^{4}, R_{7, j}^{4} !, j=1,2,3,4$, as listed in Table 3 . The group is presented as follows:

$$
\pi \Gamma_{7}^{4}(p, q, r, s)=G(-s, p,-q-r,-p)
$$

\subsection{Ribbon 2-knots obtained from $\Gamma_{8}^{4}$}

Note that $\Gamma_{8}^{4}(p, q, r, s) \stackrel{\mathrm{r}}{\sim} \Gamma_{8}^{4}(-s,-r,-q,-p)$ by the reversing move. Then we obtain the ribbon 2 -knots $R_{8,1}^{4}, R_{8,6}^{4}$, and $R_{8, j}^{4}, R_{8, j}^{4} !, j=2,3,4,5$, as listed in Table 3 . The group is presented as follows:

$$
\pi \Gamma_{8}^{4}(p, q, r, s)=G(p, s, q,-s,-p, r, p, s)
$$

\subsection{Ribbon 2-knots obtained from $\Gamma_{9}^{4}$}

If either $p=r$ or $q=s$, then $\Gamma_{9}^{4}(p, q, r, s)$ is r-equivalent to $\Gamma_{6}^{4}\left(p^{\prime}, q^{\prime}, r^{\prime}, s^{\prime}\right)$ for some signs $p^{\prime}, q^{\prime}, r^{\prime}, s^{\prime}$. In fact, if $p=r$, then by Lemma 7.2 we have $\Gamma_{9}^{4}(p, q, p, s) \stackrel{\mathrm{r}}{\sim} \Gamma_{7}^{4}(p, s, q,-s)$. Next, suppose $q=s$. By the reversing move we have $\Gamma_{9}^{4}(p, q, r, q) \stackrel{\mathrm{r}}{\sim} \Gamma_{9}^{4}(-q,-r,-q,-p)$, which is r-equivalent to $\Gamma_{6}^{4}(-q,-p,-r, p)$ by Lemma 7.2. Then we obtain the ribbon 2 -knots $R_{9,1}^{4}, R_{9,1}^{4}$ !, and $R_{9,2}^{4}$ as listed in Table 3 . The group is presented as follows:

$$
\pi \Gamma_{9}^{4}(p, q, r, s)=G(p, q,-p,-s, r, s) .
$$




\subsection{Ribbon 2-knots obtained from $\Gamma_{10}^{4}$}

From the diagram $\Gamma_{10}^{4}$ we obtain the ribbon 2 -knots $R_{10, j}^{4}, R_{10, j}^{4} !, j=1, \ldots, 8$, as listed in Table 3. The group is presented as follows:

$$
\pi \Gamma_{10}^{4}(p, q, r, s)=G(p, q,-p, r, p,-q,-p, s, p, q) .
$$

\subsection{Ribbon 2-knots obtained from $\Gamma_{11}^{4}$}

We have the following r-equivalent pairs.

\section{Lemma 7.5.}

$$
\begin{aligned}
& \Gamma_{11}^{4}(++-+) \stackrel{\mathrm{r}}{\sim} \Gamma_{11}^{4}(+-+-) \\
& \Gamma_{11}^{4}(--+-) \stackrel{\mathrm{r}}{\sim} \Gamma_{11}^{4}(-+-+) .
\end{aligned}
$$

Proof. We show Eq. (7.18). The diagram $\Gamma_{11}^{4}(++-+)$ represents the virtual arc diagram $A_{4}$ as shown in Fig. 46, which is r-equivalent to $-A_{4}^{\dagger}$. Then it is deformed into $A_{4}^{\prime}$ by the moves $\mathrm{A}_{\mathrm{II}}, \mathrm{A}_{\mathrm{III}}, \mathrm{D}, \mathrm{E}_{\mathrm{II}}$ as shown in Fig. 46 , which has the diagram $\delta_{4}$ as shown in Fig. 48.

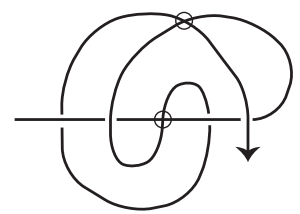

$A_{4}$

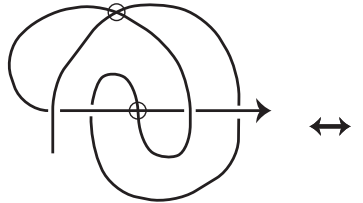

$-A_{4}^{\dagger}$
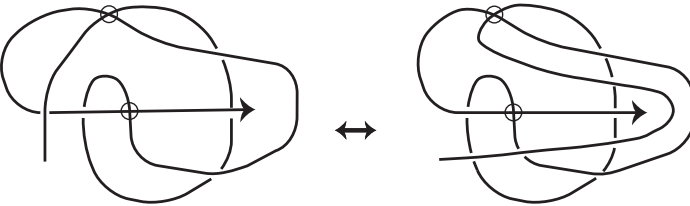

$A_{4}^{\prime}$

Fig. 46. Virtual arc diagram $A_{4}$ having diagram $\Gamma_{11}^{4}(++-+)$, and r-equivalent virtual arcs.

The diagram $\Gamma_{11}^{4}(+-+-)$ represents the virtual arc diagram $A_{5}$ as shown in Fig. 47. It is deformed into $A_{5}^{\prime}$ by the moves $\mathrm{A}_{\mathrm{II}}, \mathrm{A}_{\mathrm{III}}, \mathrm{B}_{\mathrm{II}}, \mathrm{B}_{\mathrm{III}}, \mathrm{D}, \mathrm{E}_{\mathrm{I}}, \mathrm{E}_{\mathrm{II}}$ as shown in Fig. 47, which has the Gauss diagram $\delta_{5}$ as shown in Fig. 48. Both the Gauss diagrams $\gamma_{4}$ and $\gamma_{5}$ are deformed into $\gamma_{6}$ as shown in Fig. 48 by the move D, which completes the proof.

Then we obtain the ribbon 2-knots $R_{11, j}^{4}, R_{11, j}^{4} !, j=1, \ldots, 7$, as listed in Table 3 . For the group $\pi \Gamma_{11}^{4}(p, q, r, s)$, see Example 4.4 


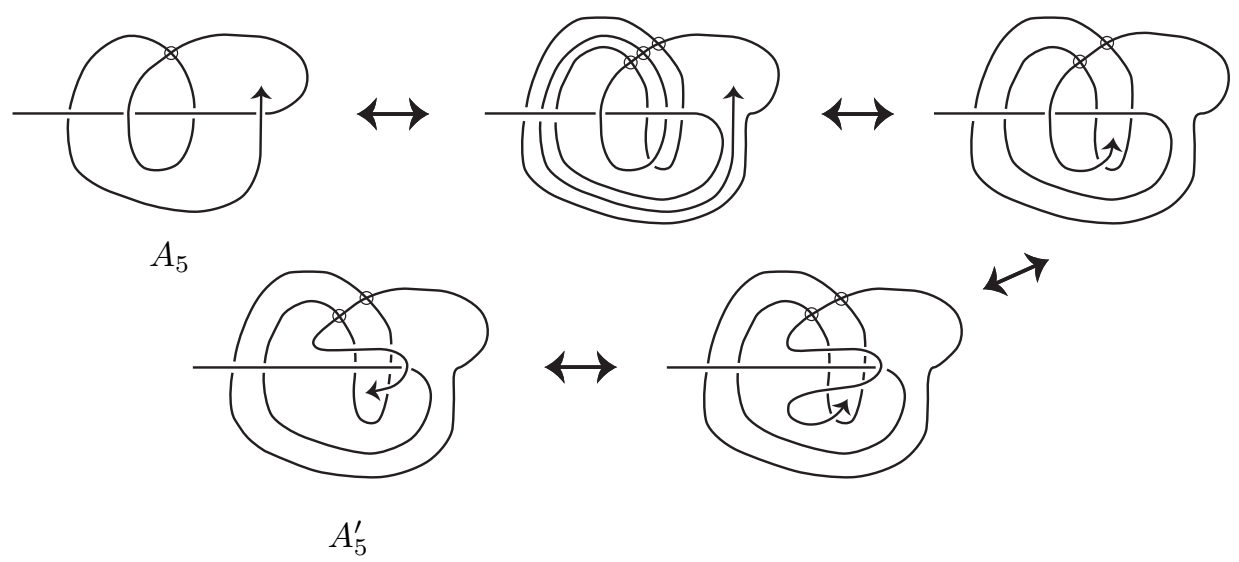

Fig. 47. Virtual arc diagram $A_{5}$ having diagram $\Gamma_{11}^{4}(+-+-)$, and r-equivalent virtual arcs.

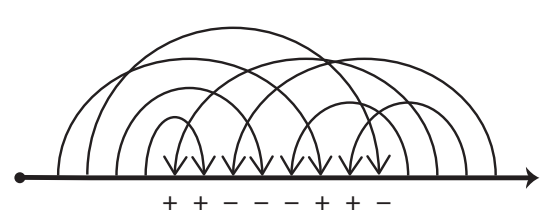

$\delta_{4}$

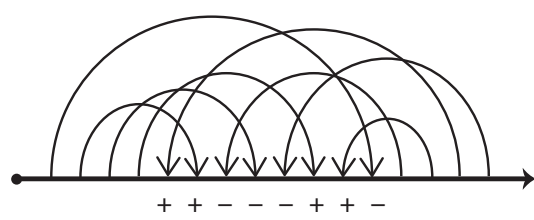

$\delta_{5}$

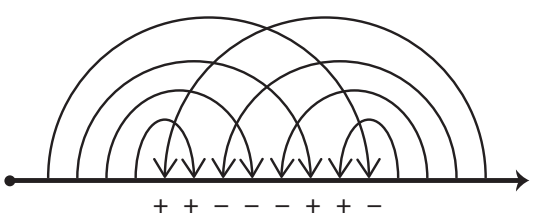

$\delta_{6}$

Fig. 48. Gauss linear diagrams for the virtual $\operatorname{arcs} A_{4}^{\prime}$ and $A_{5}^{\prime}$.

\subsection{Ribbon 2-knots obtained from $\Gamma_{12}^{4}$}

If $p=r$, then the diagram $\Gamma_{12}^{4}(p, q, r, s)$ is r-equivalent to a linear Gauss diagram with less than 4 chords. In fact, by the move $\mathrm{A}_{\mathrm{III}}$ the diagram $\Gamma_{12}^{4}(p, q, p, s)$ is r-equivalent to a linear Gauss diagram with a separated chord; see Fig. 49.

Moreover, if $s=-q$, then we have $\Gamma_{12}^{4}(p, q, r,-q) \stackrel{\mathrm{r}}{\sim}-\Gamma_{7}^{4}(p, r,-p,-q)$ as shown in Fig. 50. By the reversing move $-\Gamma_{7}^{4}(p, r,-p,-q) \stackrel{\mathrm{r}}{\sim} \Gamma_{7}^{4}(q, p,-r,-p)$. So, we consider $\Gamma_{12}^{4}(p, q, r, s)$ with $p=-r$ and $q=s$. Then we obtain the ribbon 2 -knots $R_{12, j}^{4}$, $R_{12, j}^{4} !, j=1,2$, as listed in Table 3 . The group is presented as follows:

$$
\pi \Gamma_{12}^{4}(p, q, r, s)=G(p, q, r-p, s)
$$



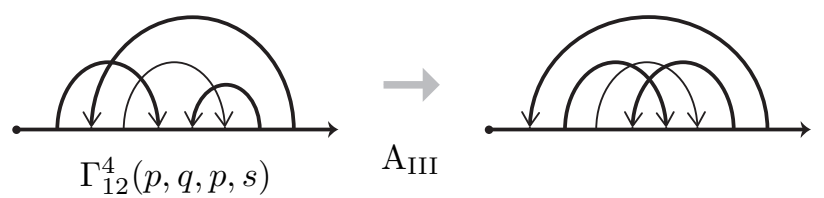

Fig. 49. $\Gamma_{12}^{4}(p, q, p, s)$ is deformed into a diagram with a single separated chord.

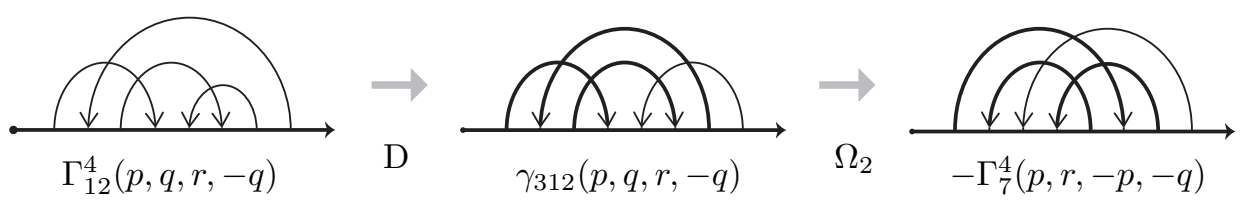

Fig. 50. $\Gamma_{12}^{4}(p, q, r,-q)$ is r-equivalent to $-\Gamma_{7}^{4}(p, r,-p,-q)$.

\subsection{Ribbon 2-knots obtained from $\Gamma_{13}^{4}$}

Since the diagram $\Gamma_{13}^{4}(p, q, r, s)$ is the product $\Gamma_{1}^{2}(p, q) \cdot \Gamma_{1}^{2}(r, s)$ and $\Gamma_{1}^{2}(++) \stackrel{\mathrm{r}}{\sim} \Gamma_{1}^{2}(--)$, by Eq. (3.1) we have:

$$
\begin{aligned}
& \left.\Gamma_{13}^{4}(++++) \stackrel{\mathrm{r}}{\sim} \Gamma_{13}^{4}(---)\right) \stackrel{\mathrm{r}}{\sim} \Gamma_{13}^{4}(++--) \stackrel{\mathrm{r}}{\sim} \Gamma_{13}^{4}(--++), \\
& \Gamma_{13}^{4}(+++-) \stackrel{\mathrm{r}}{\sim} \Gamma_{13}^{4}(+-++) \stackrel{\mathrm{r}}{\sim} \Gamma_{13}^{4}(--+-) \stackrel{\mathrm{r}}{\sim} \Gamma_{13}^{4}(+---), \\
& \Gamma_{13}^{4}(++-+) \stackrel{\mathrm{r}}{\sim} \Gamma_{13}^{4}(-+++) \stackrel{\mathrm{r}}{\sim} \Gamma_{13}^{4}(---+) \stackrel{\mathrm{r}}{\sim} \Gamma_{13}^{4}(-+--), \\
& \Gamma_{13}^{4}(+--+) \stackrel{\mathrm{r}}{\sim} \Gamma_{13}^{4}(-++-),
\end{aligned}
$$

which present the composite ribbon 2 -knots $R_{1}^{2} \# R_{1}^{2}, R_{1}^{2} \# R_{2}^{2}, R_{1}^{2} \# R_{2}^{2}$ !, $R_{2}^{2} \# R_{2}^{2}$ !, respectively. Additionally, we have $R_{2}^{2} \# R_{2}^{2}$ and $R_{2}^{2} ! \# R_{2}^{2}$ !, which are presented by $\Gamma_{13}^{4}(+-+-)$ and $\Gamma_{13}^{4}(-+-+)$, respectively. For the composition of two ribbon 2-knots $K$ and $K^{\prime}, K \# K^{\prime}$, we have $\Delta_{K \# K^{\prime}}(t)=\Delta_{K}(t) \Delta_{K^{\prime}}(t)$, and so we obtain Table 4 .

\section{Final Remarks}

(i) In Tables 2, 3 and 4 there are several pairs and triples sharing the same Alexander polynomials. Except for the pair $\left(R_{8,1}^{4}, R_{8,6}^{4}\right)$ we can distinguish using the knot group and the twisted Alexander polynomial; see the forthcoming paper $[11]$ for the detail. The groups of this pair are actually isomorphic.

(ii) The following ribbon 2-knots are missing in Yasuda's table [22,23,25,26,27]. In fact, in Yasuda's table there do not exist ribbon knots having the Alexander polynomials of these knots.

$$
R_{11,4}^{4}, R_{11,4}^{4} !, R_{11,5}^{4}, R_{11,5}^{4} !, R_{11,6}^{4}, R_{11,6}^{4} !
$$




\section{Acknowledgments}

The first author was partially supported by KAKENHI, Grant-in-Aid for Scientific Research (C) (No. 21540092), Japan Society for the Promotion of Science.

\section{References}

[1] J. Scott Carter and Masahico Saito, Knotted surfaces and their diagrams, Mathematical Surveys and Monographs, vol. 55, Amer. Math. Soc., Providence, RI, 1998.

[2] Mikhail Goussarov, Michael Polyak, and Oleg Viro, Finite-type invariants of classical and virtual knots, Topology 39 (2000), no. 5, 1045-1068.

[3] Kazuo Habiro, Taizo Kanenobu, and Akiko Shima, Finite type invariants of ribbon 2knots, in Low-dimensional topology (Funchal, 1998), Contemp. Math., vol. 233, Amer. Math. Soc., Providence, RI, 1999, pp. 187-196.

[4] Kazuo Habiro and Akiko Shima, Finite type invariants of ribbon 2-knots. II, Topology Appl. 111 (2001), no. 3, 265-287.

[5] Atsushi Ichimori and Taizo Kanenobu, Ribbon torus knots presented by virtual knots with up to four crossings, J. Knot Theory Ramifications 21 (2012), no. 13, 1240005, 30 .

[6] Taizo Kanenobu, Recursive calculation for an invariant of a ribbon knot, J. Knot Theory Ramifications 7 (1998), no. 8, 1093-1105.

[7] Taizo Kanenobu, An unknotting operation on ribbon 2-knots, J. Knot Theory Ramifications 9 (2000), no. 8, 1011-1028.

[8] Taizo Kanenobu, Forbidden moves unknot a virtual knot, J. Knot Theory Ramifications 10 (2001), no. 1, 89-96.

[9] Taizo Kanenobu, Virtual arc presentations and HC-moves of ribbon 2-knots, J. Knot Theory Ramifications 11 (2002), no. 3, 387-402, Knots 2000 Korea, Vol. 1 (Yongpyong).

[10] Taizo Kanenobu and Akiko Shima, Two filtrations of ribbon 2-knots, Topology Appl. 121 (2002), no. 1-2, 143-168.

[11] Taizo Kanenobu and Sumi Toshio, Classification of ribbon 2-knots presented by virtual arcs with up to 4 crossings, in preparation.

[12] Louis H. Kauffman, Virtual knot theory, European J. Combin. 20 (1999), no. 7, 663-690.

[13] Se-Goo Kim, Virtual knot groups and their peripheral structure, J. Knot Theory Ramifications 9 (2000), no. 6, 797-812.

[14] Seiya Komatsu, Enumeration of welded arc with up to four crossings, Master's thesis, Osaka City Universtiy, 2016 (in Japanese).

[15] Yoshihiko Marumoto, On ribbon 2-knots of 1-fusion, Math. Sem. Notes Kobe Univ. 5 (1977), no. 1, 59-68.

[16] Yoshihiko Marumoto, Stable equivalence of ribbon presentations, J. Knot Theory Ramifications 1 (1992), no. 3, 241-251.

[17] Sam Nelson, Unknotting virtual knots with Gauss diagram forbidden moves, J. Knot Theory Ramifications 10 (2001) 931-935.

[18] Michael Polyak, Minimal generating sets of Reidemeister moves, Quantum Topol. 1 (2010) 399-411.

[19] Shin Satoh, Virtual knot presentation of ribbon torus-knots, J. Knot Theory Ramifications 9 (2000) 531-542.

[20] Shin'ichi Suzuki, Knotting problems of 2-spheres in 4-sphere, Math. Sem. Notes Kobe Univ. 4 (1976) 241-371. 
[21] Takeshi Yajima, On simply knotted spheres in $R^{4}$, Osaka J. Math. 1 (1964) 133-152.

[22] Tomoyuki Yasuda, Crossing and base numbers of ribbon 2-knots, J. Knot Theory Ramifications 10 (2001) 999-1003.

[23] Tomoyuki Yasuda, Ribbon 2-knots with ribbon crossing number four, Research reports of Nara Technical College 44 (2008) 69-72 (in Japanese).

[24] Tomoyuki Yasuda, Ribbon 2-knots with distinct ribbon types, J. Knot Theory Ramifications 18 (2009) 1509-1523.

[25] Tomoyuki Yasuda, Ribbon 2-knots with ribbon crossing number four, II, Research reports of Nara Technical College 45 (2009) 59-62 (in Japanese).

[26] Tomoyuki Yasuda, Ribbon 2-knots with ribbon crossing number four, III, Research reports of Nara Technical College 46 (2010) 45-48 (in Japanese).

[27] Tomoyuki Yasuda, Ribbon 2-knots with ribbon crossing number four, IV, Research reports of Nara Technical College 47 (2011) 37-40 (in Japanese). 